\title{
Analytical solutions of bound timelike geodesic orbits in Kerr spacetime
}

\author{
Ryuichi Fujita ${ }^{1,2}$ and Wataru Hikida ${ }^{2}$ \\ ${ }^{1}$ Theoretical Physics, Raman Research Institute, Bangalore 560 080, India \\ ${ }^{2}$ Department of Earth and Space Science, Graduate School of Science, Osaka \\ University, Toyonaka, Osaka 560-0043, Japan \\ E-mail: draone@rri.res.in
}

\begin{abstract}
We derive the analytical solutions of the bound timelike geodesic orbits in Kerr spacetime. The analytical solutions are expressed in terms of the elliptic integrals using Mino time $\lambda$ as the independent variable. Mino time decouples the radial and polar motion of a particle and hence leads to forms more useful to estimate three fundamental frequencies, radial, polar and azimuthal motion, for the bound timelike geodesics in Kerr spacetime. This paper gives the first derivation of the analytical expressions of the fundamental frequencies. This paper also gives the first derivation of the analytical expressions of all coordinates for the bound timelike geodesics using Mino time. These analytical expressions should be useful not only to investigate physical properties of Kerr geodesics but more importantly to applications related to the estimation of gravitational waves from the extreme mass ratio inspirals.
\end{abstract}

PACS numbers: 04.20.Jb, 04.30.Db, 04.70.Bw, 95.30.Sf 


\section{Introduction}

The Kerr black hole has been well studied since the discovery of the Kerr solution. It is an important topic not only in mathematical problems of general theory of relativity, but also for applications in astrophysics. Currently, there are many candidates for black holes in the universe and they have a wide range of mass scales ranging from stellar mass scales to galactic nuclei mass scales [1].

One of the ways to investigate the properties of a Kerr black hole spacetime is to study geodesic motion in this background. Detailed works on the geodesic motion in black hole spacetimes are summarized in Chandrasekhar [2]. In the weak field regime, at large distances from the black hole, the orbits of a particle are almost the same as that in Newtonian gravity. In the strong field regime, however, the orbits become more complicated and it is difficult to compare the orbits with that in Newtonian gravity. For the case of bound geodesics, this can be explained by mismatches between the fundamental frequencies of radial, $\Omega_{r}$, polar, $\Omega_{\theta}$ and azimuthal-motion, $\Omega_{\phi}$. For example, $\Omega_{\phi}-\Omega_{\theta}$ shows the precession of the orbital plane and $\Omega_{\phi}-\Omega_{r}$ shows the precession of the orbital ellipse. Differences between the fundamental frequencies become larger as the particle goes into the strong gravity region around black hole horizon or separatrix, which is the boundary between stable and unstable orbits. These relativistic effects have been studied for some cases and some examples of extreme phenomena are found as follows.

Wilkins [3] derived the analytical expressions for the ratio of the azimuthal frequency and the polar frequency, $\Omega_{\phi} / \Omega_{\theta}$, when a particle moves on both circular and non-equatorial orbits around the extreme Kerr black hole. He then showed that the ratio becomes larger as the particle approaches the horizon and found that the particle traces out a helix-like orbit on a sphere around the black hole. He also pointed out that there exist horizon-skimming orbits which have the same radius as the horizon. Horizon-skimming orbits are also studied by numerical calculations including the effects of the emission of gravitational waves from a particle for circular and non-equatorial orbits [4] and for generic orbits [5] around near-extremal Kerr black holes. Glampedakis and Kennefick [6] numerically investigated the ratio of the azimuthal frequency and the radial frequency, $\Omega_{\phi} / \Omega_{r}$, when a particle moves both on eccentric and equatorial orbits around the Kerr black hole. They found that the ratio becomes larger as the particle approaches the separatrix and the particle traces out a quasi-circular orbit around the periapsis before going back to the apoapsis. These orbits are called zoom-whirl orbits.

The above results show that the fundamental frequencies play an important role in understanding bound geodesic orbits. However, the coupling of the $r$ and $\theta$ motions in the geodesic equation has prevented one from deriving the fundamental frequencies, $\Omega_{r}$, $\Omega_{\theta}$ and $\Omega_{\phi}$, for general bound geodesic orbits until recently. Using the elegant HamiltonJacobi formalism, Schmidt [7] derived the fundamental frequencies without discussing the coupling of the $r$ and $\theta$ motions. Although his results show that we can expand an arbitrary function of the particle's orbit in a Fourier series, we can not estimate the 
Fourier components because of the coupling of the $r$ and $\theta$-motion. Mino [8] showed that we can separate $r$ and $\theta$-motion if we use new time parameter $\lambda$ and derived the integral forms of the periods of both $r$ and $\theta$-motion with respect to $\lambda$, which is called Mino time. Combining Schmidt's method with Mino time, Drasco and Hughes [9] derived the fundamental frequencies and showed how the Fourier components of arbitrary functions of orbits with respect to Mino time can be computed because of the decoupling of both $r$ and $\theta$ motions. They also showed how from these results using Mino time, the Fourier components with respect to coordinate time can also be derived. Thanks to these results, one can compute gravitational waves from binary systems in which a stellar mass compact star is moving on a general bound geodesic orbit around a supermassive black hole, the so-called extreme mass ratio inspirals(EMRIs) [10]. Gravitational waves from EMRIs are one of the main targets for space-based Laser Interferometer Space Antenna (LISA) [11].

In this paper, we derive analytical expressions for bound timelike geodesic orbits in Kerr spacetime using Mino time as the independent variable. Despite a lot of works on geodesic motion 2, the analytical expressions of null or timelike geodesics in Kerr spacetime are still important subjects. Fast and accurate computation of null geodesics in Kerr spacetime is required to study radiation which pass near black holes in accretion systems such as active galactic nuclei and X-ray binaries (see, for example, [12, 13] and references therein). Fast and accurate computation of timelike geodesics is also required to study gravitational waves from EMRIs and construct efficient templates for LISA data analysis. Rauch and Blandford gave tables which reduce the some integral forms of null geodesics to Legendre elliptic integrals [14] using the radial coordinate as the independent variable [12]. They did not give the complete tables which reduce all the integral forms to the elliptic integrals because it was easier and faster to compute both $t$ and $\phi$ coordinates numerically when they studied the optical structure of the primary caustic around Kerr black hole. Using Carlson elliptic integrals [15] to calculate all coordinates of null geodesics, however, Dexter and Agol showed that they can compute null geodesics more efficiently than numerical integration method [13]. Although they did not show analytical expressions of all coordinates of null geodesics since there are so many cases to be considered, they opened their numerical code to compute null geodesics semi-analytically in Kerr spacetime. In this paper, we show that we can easily derive the analytical expressions of bound timelike geodesics in terms of Legendre elliptic integrals if we properly transform the $r$ and $\theta$ variables. This is the first time that the analytical expressions of fundamental frequencies are derived. This is also the first time that the analytical expressions of all geodesic coordinates are derived using Mino time as the independent variable. These analytical expressions of bound timelike geodesic orbits with respect to Mino time are simpler than that in [13] for null geodesics and should be useful to investigate gravitational waves from EMRIs. The analytical solutions should also be helpful for investigations of bound geodesics in Kerr spacetime.

This paper is organized as follows. In section 2, we review Kerr geodesics using observer time. We then discuss Kerr geodesics in Mino time and derive the analytical 
expressions of the fundamental frequencies of bound geodesics in section 3 . In section 4, we derive the analytical expressions for bound geodesic orbits. We conclude with a brief summary in section 5. In the Appendices, we discuss technical details of the implementation required to obtain the results in this paper. Throughout this paper, we use units with $G=c=1$.

\section{Geodesic Orbits in Kerr Spacetime}

The geodesic equations that describe a particle's orbits in Kerr spacetime are given by

$$
\begin{aligned}
& \Sigma^{2}\left(\frac{\mathrm{d} r}{\mathrm{~d} \tau}\right)^{2}=R(r), \\
& \Sigma^{2}\left(\frac{\mathrm{d} \cos \theta}{\mathrm{d} \tau}\right)^{2}=\Theta(\cos \theta), \\
& \Sigma \frac{\mathrm{d} t}{\mathrm{~d} \tau}=T_{\mathrm{r}}(r)+T_{\theta}(\cos \theta)+a \mathcal{L}_{z}, \\
& \Sigma \frac{\mathrm{d} \phi}{\mathrm{d} \tau}=\Phi_{\mathrm{r}}(r)+\Phi_{\theta}(\cos \theta)-a \mathcal{E} .
\end{aligned}
$$

The functions $R(r), \Theta(\cos \theta), T_{\mathrm{r}}(r), T_{\theta}(\cos \theta), \Phi_{\mathrm{r}}(r)$ and $\Phi_{\theta}(\cos \theta)$ are defined by

$$
\begin{aligned}
& R(r)=[P(r)]^{2}-\Delta\left[r^{2}+\left(a \mathcal{E}-\mathcal{L}_{z}\right)^{2}+\mathcal{C}\right], \\
& \Theta(\cos \theta)=\mathcal{C}-\left(\mathcal{C}+a^{2}\left(1-\mathcal{E}^{2}\right)+\mathcal{L}_{z}^{2}\right) \cos ^{2} \theta+a^{2}\left(1-\mathcal{E}^{2}\right) \cos ^{4} \theta, \\
& T_{r}(r)=\frac{r^{2}+a^{2}}{\Delta} P(r), \quad T_{\theta}(\cos \theta)=-a^{2} \mathcal{E}\left(1-\cos ^{2} \theta\right), \\
& \Phi_{r}(r)=\frac{a}{\Delta} P(r), \quad \Phi_{\theta}(\cos \theta)=\frac{\mathcal{L}_{z}}{1-\cos ^{2} \theta},
\end{aligned}
$$

with $P(r)=\mathcal{E}\left(r^{2}+a^{2}\right)-a \mathcal{L}_{z}, \Sigma=r^{2}+a^{2} \cos ^{2} \theta$ and $\Delta=r^{2}-2 M r+a^{2}$. Here $M$ and $a$ are the mass and the angular momentum of the black hole, respectively. There are three constants of motion, $\mathcal{E}, \mathcal{L}_{z}$ and $\mathcal{C}$, which are the energy, the z-component of the angular momentum and the Carter constant per unit mass, respectively. Using reasonable initial conditions for the particle's orbit, we can derive the orbits using the proper time of the particle, $\tau$, by numerical integration. Dividing $\mathrm{d} r / \mathrm{d} \tau, \mathrm{d} \cos \theta / \mathrm{d} \tau$ and $\mathrm{d} \phi / \mathrm{d} \tau$ by $\mathrm{d} t / \mathrm{d} \tau$, we can also derive the orbits with coordinate time $t$ by numerical integration. When the orbits are bound to black hole, however, we have to take care of the turning points in the radial and the polar motion where the signs of $\mathrm{d} r / \mathrm{d} \tau$ and $\mathrm{d} \cos \theta / \mathrm{d} \tau$ change. These turning points correspond to periapsis and apoapsis for the radial motion, and $\theta_{\min }$ and $\pi-\theta_{\min }$ for the polar motion, where $\theta_{\min }$ is the minimum value of $\theta$. We need smaller stepsizes to resolve the derivatives around turning points. We can avoid this problem by introducing new variables for the radial and polar motion, $r=p M /(1+e \cos \psi)$ and $\cos \theta=\cos \theta_{\min } \cos \chi$, where $p$ is semilatus rectum and $e$ is eccentricity [9]. Using these new variables, $\psi$ and $\chi$, we can estimate the orbits accurately.

There exists three fundamental frequencies, $\Omega_{r}, \Omega_{\theta}$ and $\Omega_{\phi}$, for bound Kerr geodesics. However, it is difficult to estimate the fundamental frequencies using (1) because of the coupling of the $r$ and $\theta$-motions. For instance, we immediately face 
a difficulty when we estimate $\Omega_{r}$ using $\mathrm{d} r / \mathrm{d} t=(\mathrm{d} r / \mathrm{d} \tau)(\mathrm{d} t / \mathrm{d} \tau)^{-1}$ because $r$ and $\theta$ asynchronously pass their turning points.

\section{Fundamental Frequencies of bound geodesics}

We now proceed to derive the analytical expressions for the fundamental frequencies of bound geodesic orbits using Mino time. In section 3.1, we briefly describe the Kerr geodesics in Mino time and then show how to derive the analytical expressions for the fundamental frequencies in section 3.2 and section 3.3. In section 3.4, we will check the analytical expressions by comparing them with earlier literature.

\subsection{Geodesics in Mino time}

Using Mino time, $\lambda=\int \mathrm{d} \tau / \Sigma$, the geodesic equations become

$$
\begin{aligned}
& \left(\frac{\mathrm{d} r}{\mathrm{~d} \lambda}\right)^{2}=R(r), \\
& \left(\frac{\mathrm{d} \cos \theta}{\mathrm{d} \lambda}\right)^{2}=\Theta(\cos \theta), \\
& \frac{\mathrm{d} t}{\mathrm{~d} \lambda}=T_{\mathrm{r}}(r)+T_{\theta}(\cos \theta)+a \mathcal{L}_{z}, \\
& \frac{\mathrm{d} \phi}{\mathrm{d} \lambda}=\Phi_{\mathrm{r}}(r)+\Phi_{\theta}(\cos \theta)-a \mathcal{E} .
\end{aligned}
$$

It should be noted that, in (2), $\mathrm{d} r / \mathrm{d} \lambda$ depends only on $r$ and $\mathrm{d} \cos \theta / \mathrm{d} \lambda$ depends only on $\cos \theta$. Thus the equations for radial and polar motion are decoupled. For the bound orbits, $r(\lambda)$ and $\cos \theta(\lambda)$ become periodic functions which are independent of each other. The fundamental periods for the radial and polar motion, $\Lambda_{r}$ and $\Lambda_{\theta}$, with respect to $\lambda$ are given by

$$
\Lambda_{r}=2 \int_{\mathrm{r}_{\min }}^{\mathrm{r}_{\max }} \frac{\mathrm{dr}}{\sqrt{R(r)}}, \quad \Lambda_{\theta}=4 \int_{0}^{\cos \theta_{\min }} \frac{\mathrm{d} \cos \theta}{\sqrt{\Theta(\cos \theta)}},
$$

where

$$
r_{\min }=\frac{p M}{1+e}, \quad r_{\max }=\frac{p M}{1-e}, \quad \theta_{\mathrm{inc}}+\left(\operatorname{sgn} \mathcal{L}_{z}\right) \theta_{\min }=\frac{\pi}{2} .
$$

Here $r_{\min }$ and $r_{\max }$ are the periapsis and apoapsis for the radial motion respectively, and $\theta_{\text {inc }}$ the inclination angle from the equatorial plane of black hole. Of course, $\left(\mathcal{E}, \mathcal{L}_{z}, \mathcal{C}\right)$ are described by these orbital parameters $\left(p, e, \theta_{\text {inc }}\right)$ and given in [7, 9]. The angular frequencies of the radial and the polar motion then become

$$
\Upsilon_{r}=\frac{2 \pi}{\Lambda_{r}}, \quad \Upsilon_{\theta}=\frac{2 \pi}{\Lambda_{\theta}}
$$

We also note that both $\mathrm{d} t / \mathrm{d} \lambda$ and $\mathrm{d} \phi / \mathrm{d} \lambda$ in (2) are the sum of a function of $r$ and a function of $\cos \theta$. Then each equations are integrated as

$$
t(\lambda)=\Gamma \lambda+t^{(r)}(\lambda)+t^{(\theta)}(\lambda), \quad \phi(\lambda)=\Upsilon_{\phi} \lambda+\phi^{(r)}(\lambda)+\phi^{(\theta)}(\lambda),
$$


where $\Gamma$ and $\Upsilon_{\phi}$ are the frequencies of coordinate time $t$ and $\phi$ with respect to $\lambda$ respectively, which are given by

$$
\begin{array}{ll}
\Gamma=\Upsilon_{t^{(r)}}+\Upsilon_{t^{(\theta)}}+a \mathcal{L}_{z}, & \Upsilon_{\phi}=\Upsilon_{\phi^{(r)}}+\Upsilon_{\phi^{(\theta)}}-a \mathcal{E}, \\
\Upsilon_{t^{(r)}}=\left\langle T_{r}(r)\right\rangle_{\lambda}, & \Upsilon_{t^{(\theta)}}=\left\langle T_{\theta}(\cos \theta)\right\rangle_{\lambda}, \\
\Upsilon_{\phi^{(r)}}=\left\langle\Phi_{r}(r)\right\rangle_{\lambda}, & \Upsilon_{\phi^{(\theta)}}=\left\langle\Phi_{\theta}(\cos \theta)\right\rangle_{\lambda},
\end{array}
$$

where $\langle\cdots\rangle_{\lambda} \equiv \lim _{\Delta \lambda \rightarrow \infty}(2 \Delta \lambda)^{-1} \int_{-\Delta \lambda}^{\Delta \lambda} d \lambda \cdots$ represents infinite time average with respect to $\lambda$, and $t^{(r) /(\theta)}$ and $\phi^{(r) /(\theta)}$ satisfy

$$
\begin{array}{ll}
\frac{\mathrm{d} t^{(r)}}{\mathrm{d} \lambda}=T_{r}(r)-\Upsilon_{t^{(r)}}, & \frac{\mathrm{d} t^{(\theta)}}{\mathrm{d} \lambda}=T_{\theta}(\cos \theta)-\Upsilon_{t^{(\theta)}}, \\
\frac{\mathrm{d} \phi^{(r)}}{\mathrm{d} \lambda}=\Phi_{r}(r)-\Upsilon_{\phi^{(r)}}, & \frac{\mathrm{d} \phi^{(\theta)}}{\mathrm{d} \lambda}=\Phi_{\theta}(\cos \theta)-\Upsilon_{\phi^{(\theta)}} .
\end{array}
$$

Equation (6) shows that both $t(\lambda)$ and $\phi(\lambda)$ consist of two distinct parts. The first term represents an accumulation over $\lambda$-time and the last two terms represent oscillations around it with periods $2 \pi / \Upsilon_{r}$ and $2 \pi / \Upsilon_{\theta}$. We note that the frequencies with respect to $\lambda$ are related to the frequencies with distant observer time as [9]

$$
\Omega_{r}=\frac{\Upsilon_{r}}{\Gamma}, \quad \Omega_{\theta}=\frac{\Upsilon_{\theta}}{\Gamma}, \quad \Omega_{\phi}=\frac{\Upsilon_{\phi}}{\Gamma} .
$$

In the following subsections, section 3.2 and section 3.3 , we discuss the analytical expressions for these frequencies. And we discuss the analytical expressions of the orbits, $r(\lambda), \cos \theta(\lambda), t(\lambda)$ and $\phi(\lambda)$, in section 4 .

\subsection{Frequencies of $r$ and $\theta$-motion}

In this subsection, we derive the analytical expressions for the frequencies of $r$ and $\theta$ motion, $\Upsilon_{r}$ and $\Upsilon_{\theta}$, using (3) . As explained in section 2 , $R(r)$ and $\Theta(\cos \theta)$ become zero when $r$ and $\cos \theta$ go through the turning points, $r_{\min }, r_{\max }$ and $\pm \cos \theta_{\min }$, respectively. Thus we usually transform the variables, $r$ and $\cos \theta$, to avoid divergences in the numerical calculation. However we know that (3) can be expressed in terms of the elliptic integrals since both $R(r)$ and $\Theta(\cos \theta)$ are fourth order polynomials [16]. It is useful if we know the four zero points of both $R(r)$ and $\Theta(\cos \theta)$ to express (3) in terms of the elliptic integrals. We rewrite $R(r)$ and $\Theta(\cos \theta)$ as [9]

$$
\begin{aligned}
& R(r)=\left(1-\mathcal{E}^{2}\right)\left(r_{1}-r\right)\left(r-r_{2}\right)\left(r-r_{3}\right)\left(r-r_{4}\right), \\
& \Theta(\cos \theta)=\mathcal{L}_{z}^{2} \epsilon_{0}\left(z_{-}-\cos ^{2} \theta\right)\left(z_{+}-\cos ^{2} \theta\right),
\end{aligned}
$$

where

$$
\begin{aligned}
r_{1}=\frac{p M}{1-e}, \quad r_{2} & =\frac{p M}{1+e}, \quad r_{3}=\frac{(A+B)+\sqrt{(A+B)^{2}-4 A B}}{2}, \quad r_{4}=\frac{A B}{r_{3}}, \\
A+B & =\frac{2 M}{1-\mathcal{E}^{2}}-\left(r_{1}+r_{2}\right), \quad A B=\frac{a^{2} \mathcal{C}}{\left(1-\mathcal{E}^{2}\right) r_{1} r_{2}},
\end{aligned}
$$

and where $\epsilon_{0}=a^{2}\left(1-\mathcal{E}^{2}\right) / \mathcal{L}_{z}^{2}, z_{-}=\cos ^{2} \theta_{\min }$ and $z_{+}=\mathcal{C} /\left(\mathcal{L}_{z}^{2} \epsilon_{0} z_{-}\right)$. We note that two zero points, $r_{1}$ and $r_{2}$, of $R(r)$ are apoapsis and periapis respectively and two zero points, $z_{-}$and $-z_{-}$, of $\Theta(\cos \theta)$ are $\theta_{\min }$ and $\pi-\theta_{\min }$ respectively. These zero points 
correspond to turning points, defined in (4), of radial and polar motion. But the other two zero points of both $R(r)$ and $\Theta(\cos \theta), r_{3}, r_{4}$ and $\pm z_{+}$, do not correspond to turning points of radial and polar motion.

Using (10), we can express (31) in terms of the elliptic integrals as

$$
\begin{aligned}
& \int_{r_{2}}^{r} \frac{\mathrm{d} r^{\prime}}{\sqrt{R\left(r^{\prime}\right)}}=\frac{2}{\sqrt{\left(1-\mathcal{E}^{2}\right)\left(r_{1}-r_{3}\right)\left(r_{2}-r_{4}\right)}} F\left(\arcsin y_{r}, k_{r}\right), \\
& \int_{0}^{\cos \theta} \frac{\mathrm{d} \cos \theta^{\prime}}{\sqrt{\Theta\left(\cos \theta^{\prime}\right)}}=\frac{1}{\mathcal{L}_{z} \sqrt{\epsilon_{0} z_{+}}} F\left(\arcsin y_{\theta}, k_{\theta}\right),
\end{aligned}
$$

where

$$
\begin{aligned}
& y_{r}=\sqrt{\frac{r_{1}-r_{3}}{r_{1}-r_{2}} \frac{r-r_{2}}{r-r_{3}}}, \quad k_{r}=\sqrt{\frac{r_{1}-r_{2}}{r_{1}-r_{3}} \frac{r_{3}-r_{4}}{r_{2}-r_{4}}}, \\
& y_{\theta}=\frac{\cos \theta}{\sqrt{z_{-}}}, \quad k_{\theta}=\sqrt{\frac{z_{-}}{z_{+}}},
\end{aligned}
$$

and $F(\varphi, k)$ is the incomplete elliptic integral of the first kind defined by

$$
F(\varphi, k)=\int_{0}^{\varphi} \frac{\mathrm{d} \theta}{\sqrt{1-k^{2} \sin ^{2} \theta}}=\int_{0}^{\sin \varphi} \frac{\mathrm{d} y}{\sqrt{\left(1-y^{2}\right)\left(1-k^{2} y^{2}\right)}} .
$$

In the following, we describe both the elliptic integrals and the elliptic functions using the notation in [17]. The orbital frequencies of radial and polar motion with respect to $\lambda$ are then given by

$$
\Upsilon_{r}=\frac{\pi \sqrt{\left(1-\mathcal{E}^{2}\right)\left(r_{1}-r_{3}\right)\left(r_{2}-r_{4}\right)}}{2 K\left(k_{r}\right)}, \quad \Upsilon_{\theta}=\frac{\pi \mathcal{L}_{z} \sqrt{\epsilon_{0} z_{+}}}{2 K\left(k_{\theta}\right)}
$$

Here $K(k)$ is the complete elliptic integral of the first kind defined by $K(k)=F(\pi / 2, k)$. We note that the analysis of $\theta$-motion here is similar to that of Drasco and Hughes [9]. Though they used a different transformation of $\cos \theta$, our expression for $\Upsilon_{\theta}$ in this subsection agrees with their final result.

\subsection{Frequencies of $t$ and $\phi$-motion}

In this subsection, we derive the analytical expressions for the frequencies of $t$ and $\phi$ motion, $\Gamma$ and $\Upsilon_{\phi}$, using (7). Since the $r$ and $\theta$-motion decouple in Mino time, we can rewrite the infinite time average in (77) as an average over an orbital period, $\Lambda_{r}$ or $\Lambda_{\theta}$, as

$$
\begin{array}{ll}
\Upsilon_{t^{(r)}}=\frac{2}{\Lambda_{r}} \int_{r_{2}}^{r_{1}} \frac{T_{r}(r)}{\sqrt{R(r)}} \mathrm{d} r, & \Upsilon_{t^{(\theta)}}=\frac{4}{\Lambda_{\theta}} \int_{0}^{\sqrt{z_{-}}} \frac{T_{\theta}(\cos \theta)}{\sqrt{\Theta(\cos \theta)} \mathrm{d} \cos \theta,} \\
\Upsilon_{\phi^{(r)}}=\frac{2}{\Lambda_{r}} \int_{r_{2}}^{r_{1}} \frac{\Phi_{r}(r)}{\sqrt{R(r)}} \mathrm{d} r, & \Upsilon_{\phi^{(\theta)}}=\frac{4}{\Lambda_{\theta}} \int_{0}^{\sqrt{z_{-}}} \frac{\Phi_{\theta}(\cos \theta)}{\sqrt{\Theta(\cos \theta)}} \mathrm{d} \cos \theta .
\end{array}
$$

It is straightforward to express $\Upsilon_{t^{(\theta)}}$ and $\Upsilon_{\phi^{(\theta)}}$ in terms of the elliptic integrals if we use $y_{\theta}$ in $(\underline{13})$.

$$
\Upsilon_{t^{(\theta)}}=-\frac{2 a^{2} \mathcal{E} \Upsilon_{\theta}}{\pi \mathcal{L}_{z} \sqrt{\epsilon_{0} z_{+}}}\left[\left(1-z_{+}\right) K\left(k_{\theta}\right)+z_{+} E\left(\frac{\pi}{2}, k_{\theta}\right)\right]
$$


Analytical solutions of bound timelike geodesic orbits in Kerr spacetime

$$
\Upsilon_{\phi^{(\theta)}}=\frac{2 \Upsilon_{\theta}}{\pi \sqrt{\epsilon_{0} z_{+}}} \Pi\left(\frac{\pi}{2},-z_{-}, k_{\theta}\right)
$$

where $E(\varphi, k)$ is the incomplete elliptic integral of the second kind and $\Pi(\varphi, c, k)$ is the incomplete elliptic integral of the third kind defined by

$$
\begin{aligned}
& E(\varphi, k)=\int_{0}^{\varphi} \sqrt{1-k^{2} \sin ^{2} \theta} \mathrm{d} \theta, \\
& \Pi(\varphi, c, k)=\int_{0}^{\varphi} \frac{\mathrm{d} \theta}{\left(1+c \sin ^{2} \theta\right) \sqrt{1-k^{2} \sin ^{2} \theta}} .
\end{aligned}
$$

Note that, $E(\pi / 2, k)$ is the complete elliptic integral of the second kind and $\Pi(\pi / 2, c, k)$ is the complete elliptic integral of the third kind. In the followings, we describe $E(\pi / 2, k)$ as $E(k)$, and $\Pi(\pi / 2, c, k)$ as $\Pi(c, k)$.

On the other hand, we have to rewrite $T_{r}(r)$ and $\Phi_{r}(r)$ in order to express $\Upsilon_{t^{(r)}}$ and $\Upsilon_{\phi^{(r)}}$ in terms of the elliptic integrals. Performing partial fraction decomposition, we decompose $T_{r}(r)$ and $\Phi_{r}(r)$ as follows.

$$
\begin{aligned}
T_{r}(r)= & \mathcal{E}^{2}+2 M \mathcal{E} r+\frac{2 M}{r_{+}-r_{-}}\left\{\frac{\left(4 M^{2} \mathcal{E}-a \mathcal{L}_{z}\right) r_{+}-2 M a^{2} \mathcal{E}}{r-r_{+}}-(+\leftrightarrow-)\right\} \\
& +\left(a^{2}+4 M^{2}\right) \mathcal{E}-a \mathcal{L}_{z}(|a| \neq M), \\
= & \mathcal{E} r^{2}+2 M \mathcal{E} r+\frac{2 M\left(4 M^{2} \mathcal{E}-a \mathcal{L}_{z}\right)}{r-M}+\frac{2 M^{2}\left(2 M^{2} \mathcal{E}-a \mathcal{L}_{z}\right)}{(r-M)^{2}} \\
& +\left(a^{2}+4 M^{2}\right) \mathcal{E}-a \mathcal{L}_{z} \quad(|a|=M), \\
\Phi_{r}(r)= & \frac{a}{r_{+}-r_{-}}\left\{\frac{2 M \mathcal{E} r_{+}-a \mathcal{L}_{z}}{r-r_{+}}-(+\leftrightarrow-)\right\}+a \mathcal{E} \quad(|a| \neq M), \\
= & \frac{2 M a \mathcal{E}}{r-M}+\frac{a\left(2 M^{2} \mathcal{E}-a \mathcal{L}_{z}\right)}{(r-M)^{2}}+a \mathcal{E} \quad(|a|=M),
\end{aligned}
$$

with $r_{ \pm}=M \pm \sqrt{M^{2}-a^{2}}$. From (13), we find $r=r_{3}+\left(r_{2}-r_{3}\right) /\left(1-h_{r} y_{r}^{2}\right)$, where $h_{r}=\left(r_{1}-r_{2}\right) /\left(r_{1}-r_{3}\right)$. Then it is straightforward to compute $\int_{r_{2}}^{r} r^{\prime} \mathrm{d} r^{\prime} / \sqrt{R\left(r^{\prime}\right)}$, which is composed in $\Upsilon_{t^{(r)}}$, as

$$
\left.\int_{r_{2}}^{r} \frac{r^{\prime}}{\sqrt{R\left(r^{\prime}\right)}} \mathrm{d} r^{\prime}=\frac{2\left[r_{3} F\left(\arcsin y_{r}, k_{r}\right)+\left(r_{2}-r_{3}\right) \Pi\left(\arcsin y_{r},-h_{r}, k_{r}\right)\right]}{\sqrt{\left(1-\mathcal{E}^{2}\right)\left(r_{1}-r_{3}\right)\left(r_{2}-r_{4}\right)}} .20\right)
$$

We show the results of the other terms of $\int_{r_{2}}^{r} T_{r}\left(r^{\prime}\right) \mathrm{d} r^{\prime} / \sqrt{R\left(r^{\prime}\right)}$ and $\int_{r_{2}}^{r} \Phi_{r}\left(r^{\prime}\right) \mathrm{d} r^{\prime} / \sqrt{R\left(r^{\prime}\right)}$ in Appendix A. Using results quoted there, we derive $\Gamma$ and $\Upsilon_{\phi}$ ast

$$
\begin{aligned}
\Gamma=4 M^{2} \mathcal{E}+ & \frac{2 a^{2} \mathcal{E} z_{+} \Upsilon_{\theta}}{\pi \mathcal{L}_{z} \sqrt{\epsilon_{0} z_{+}}}\left[K\left(k_{\theta}\right)-E\left(k_{\theta}\right)\right]+\frac{2 \Upsilon_{r}}{\pi \sqrt{\left(1-\mathcal{E}^{2}\right)\left(r_{1}-r_{3}\right)\left(r_{2}-r_{4}\right)}} \\
& \times\left\{\frac { \mathcal { E } } { 2 } \left[\left(r_{3}\left(r_{1}+r_{2}+r_{3}\right)-r_{1} r_{2}\right) K\left(k_{r}\right)\right.\right. \\
& +\left(r_{2}-r_{3}\right)\left(r_{1}+r_{2}+r_{3}+r_{4}\right) \Pi\left(-h_{r}, k_{r}\right) \\
& \left.+\left(r_{1}-r_{3}\right)\left(r_{2}-r_{4}\right) E\left(k_{r}\right)\right]+2 M \mathcal{E}\left[r_{3} K\left(k_{r}\right)+\left(r_{2}-r_{3}\right) \Pi\left(-h_{r}, k_{r}\right)\right] \\
& +\frac{2 M}{r_{+}-r_{-}}\left[\frac{\left(4 M^{2} \mathcal{E}-a \mathcal{L}_{z}\right) r_{+}-2 M a^{2} \mathcal{E}}{r_{3}-r_{+}}\left(K\left(k_{r}\right)-\frac{r_{2}-r_{3}}{r_{2}-r_{+}} \Pi\left(-h_{+}, k_{r}\right)\right)\right.
\end{aligned}
$$

$\ddagger$ As pointed out in [18], in the first version of this article there was a typo in $\Gamma$ : the square closing bracket in the third line should be moved to include the next term, i.e. $\left(r_{1}-r_{3}\right)\left(r_{2}-r_{4}\right) E\left(k_{r}\right)$. We thank C. F. Sopuerta and N. Yunes for pointing out the typo. 


$$
\begin{aligned}
& -(+\leftrightarrow-)]\}, \\
\Upsilon_{\phi}=\frac{2 \Upsilon_{\theta}}{\pi \sqrt{\epsilon_{0} z_{+}}} & \Pi\left(-z_{-}, k_{\theta}\right)+\frac{2 a \Upsilon_{r}}{\pi\left(r_{+}-r_{-}\right) \sqrt{\left(1-\mathcal{E}^{2}\right)\left(r_{1}-r_{3}\right)\left(r_{2}-r_{4}\right)}} \\
& \times\left\{\frac{2 M \mathcal{E} r_{+}-a \mathcal{L}_{z}}{r_{3}-r_{+}}\left[K\left(k_{r}\right)-\frac{r_{2}-r_{3}}{r_{2}-r_{+}} \Pi\left(-h_{+}, k_{r}\right)\right]-(+\leftrightarrow-)\right\},
\end{aligned}
$$

where $h_{ \pm}=\left(r_{1}-r_{2}\right)\left(r_{3}-r_{ \pm}\right) /\left[\left(r_{1}-r_{3}\right)\left(r_{2}-r_{ \pm}\right)\right]$. Combining (91), (15) and (21), we can derive the orbital frequencies with respect to observer time, $\Omega_{r}, \Omega_{\theta}$ and $\Omega_{\phi}$. It should be noted that (21) is not valid for the case $|a|=M$ since there exists divergent terms in $1 /\left(r_{+}-r_{-}\right)$. We show the expressions of $\Gamma$ and $\Upsilon_{\phi}$ for the case $|a|=M$ in Appendix B.

\subsection{Consistency check of the fundamental frequencies}

We can compare the expressions for the fundamental frequencies, $\Omega_{r}, \Omega_{\theta}$ and $\Omega_{\phi}$, in this section with that in earlier literature for some limiting cases. We can compare $\Omega_{\phi}$ for the case $e=0$ and $\theta_{\text {inc }}=0$ with [19], $\Omega_{\phi} / \Omega_{r}$ in the case $a=0, e \neq 0$ and $\theta_{\text {inc }}=0$ with [20], and both $\Omega_{\phi}$ and $\Omega_{\theta}$ in the case $e=0$ and $\theta_{\text {inc }} \neq 0$ with [21]. It is a good check on our results that our more general analytical expressions for the fundamental frequencies are consistent with earlier work for the limiting cases.

We can check our results for more general cases. In table 1 and table 2, we compare our results with [22] in which the analytical expressions of the orbital frequencies are derived in terms of both post-Newtonian and small eccentricity expansions up through $O\left(v^{5}, e^{2}\right)$, where $v^{2}=M / p$. In table 1 and table 2, we check our results for the cases $a \neq M$ and $a=M$ respectively. We find that relative errors are always less than $10^{-4}$ when we compare the results for the cases $p=100 M$ and $0.01 \leq e \leq 0.09$. Since the fundamental frequencies in [22] are derived up through $O\left(v^{5}, e^{2}\right)$, these relative errors, less than $10^{-4}$, show the consistency of our results with [22]. In table 3, we compare our results with numerical integration method for the eccentric and inclined orbits such that $p=6 M, e=0.7, \theta_{\text {inc }}=20^{\circ}$ and $a=0.9 M$ or $a=M$. In numerical integration method, we use the trapezium rule to compute the fundamental frequencies. We can compute very accurately if we use the trapezium rule for the numerical integration of a periodic function. Then we find that the analytical expressions of the fundamental frequencies in this section agree with the results of numerical integration method. The relative errors are less than $10^{-15}$ in double precision calculation. These facts show that the analytical expressions in this section are correct in the cases of generic bound orbits.

\section{Analytical solutions of bound geodesics}

In this section, we derive the analytical expressions for bound geodesic orbits, $r(\lambda)$, $\cos \theta(\lambda), t(\lambda)$ and $\phi(\lambda)$, in terms of the elliptic integrals. Since we have already derived the orbital frequencies in terms of the complete elliptic integrals in section 3, we can derive the orbits if we replace the complete elliptic integrals with the incomplete elliptic integrals. However, we have to take account of the initial values of both $r$ and $\theta$ and 
Table 1. Comparison of the orbital frequencies, $\Omega_{r}, \Omega_{\theta}$ and $\Omega_{\phi}$, derived using analytical expressions in this work and the analytical post-Newtonian expressions for orbits which are slightly eccentric but greatly inclined [22] in the case of $a=0.9 M$ and $p=100 M$. Our results are consistent with post-Newtonian results. Relative errors of the orbital frequencies are always less than $10^{-4}$.

\begin{tabular}{|c|c|c|c|c|c|c|c|}
\hline$e$ & $\theta_{\text {inc }}$ & $\Omega_{r}^{\text {This work }}$ & $\Omega_{\theta}^{\text {This work }}$ & $\Omega_{\phi}^{\text {This work }}$ & $\Omega_{r}^{\text {Post-Newton }}$ & $\Omega_{\theta}^{\text {Post-Newton }}$ & $\Omega_{\phi}^{\text {Post-Newton }}$ \\
\hline 0.01 & $20^{\circ}$ & $9.71944 \times 10^{-4}$ & $9.97417 \times 10^{-4}$ & $9.99102 \times 10^{-4}$ & $9.71979 \times 10^{-4}$ & $9.97416 \times 10^{-4}$ & $9.99102 \times 10^{-4}$ \\
\hline 0.01 & $45^{\circ}$ & $9.71338 \times 10^{-4}$ & $9.97974 \times 10^{-4}$ & $9.99688 \times 10^{-4}$ & $9.71367 \times 10^{-4}$ & $9.97975 \times 10^{-4}$ & $9.99689 \times 10^{-4}$ \\
\hline 0.01 & $70^{\circ}$ & $9.70356 \times 10^{-4}$ & $9.98889 \times 10^{-4}$ & $1.00065 \times 10^{-3}$ & $9.70379 \times 10^{-4}$ & $9.98891 \times 10^{-4}$ & $1.00065 \times 10^{-3}$ \\
\hline 0.05 & $20^{\circ}$ & $9.68513 \times 10^{-4}$ & $9.93895 \times 10^{-4}$ & $9.95575 \times 10^{-4}$ & $9.68558 \times 10^{-4}$ & $9.93905 \times 10^{-4}$ & $9.95586 \times 10^{-4}$ \\
\hline 0.05 & $45^{\circ}$ & $9.67910 \times 10^{-4}$ & $9.94452 \times 10^{-4}$ & $9.96160 \times 10^{-4}$ & $9.67949 \times 10^{-4}$ & $9.94464 \times 10^{-4}$ & $9.96173 \times 10^{-4}$ \\
\hline 0.05 & $70^{\circ}$ & $9.66934 \times 10^{-4}$ & $9.95366 \times 10^{-4}$ & $9.97118 \times 10^{-4}$ & $9.66968 \times 10^{-4}$ & $9.95380 \times 10^{-4}$ & $9.97132 \times 10^{-4}$ \\
\hline 0.09 & $20^{\circ}$ & $9.60520 \times 10^{-4}$ & $9.85694 \times 10^{-4}$ & $9.87360 \times 10^{-4}$ & $9.60669 \times 10^{-4}$ & $9.85810 \times 10^{-4}$ & $9.87478 \times 10^{-4}$ \\
\hline 0.09 & $45^{\circ}$ & $9.59926 \times 10^{-4}$ & $9.86249 \times 10^{-4}$ & $9.87943 \times 10^{-4}$ & $9.60069 \times 10^{-4}$ & $9.86368 \times 10^{-4}$ & $9.88063 \times 10^{-4}$ \\
\hline 0.09 & $70^{\circ}$ & $9.58963 \times 10^{-4}$ & $9.87162 \times 10^{-4}$ & $9.88899 \times 10^{-4}$ & $9.59101 \times 10^{-4}$ & $9.87282 \times 10^{-4}$ & $9.89020 \times 10^{-4}$ \\
\hline
\end{tabular}

Table 2. Comparison of the orbital frequencies, $\Omega_{r}, \Omega_{\theta}$ and $\Omega_{\phi}$, derived using analytical expressions in this work and the analytical post-Newtonian expressions for orbits which are slightly eccentric but greatly inclined 22 in the case of $a=M$ and $p=100 M$. Our results are consistent with post-Newtonian results. Relative errors of the orbital frequencies are always less than $10^{-4}$.

\begin{tabular}{|c|c|c|c|c|c|c|c|}
\hline$e$ & $\theta_{\text {inc }}$ & $\Omega_{r}^{\text {This work }}$ & $\Omega_{\theta}^{\text {This work }}$ & $\Omega_{\phi}^{\text {This work }}$ & $\Omega_{r}^{\text {Post-Newton }}$ & $\Omega_{\theta}^{\text {Post-Newton }}$ & $\Omega_{\phi}^{\text {Post-Newton }}$ \\
\hline 0.01 & $20^{\circ}$ & $9.72213 \times 10^{-4}$ & $9.97159 \times 10^{-4}$ & $9.99017 \times 10^{-4}$ & $9.72250 \times 10^{-4}$ & $9.97157 \times 10^{-4}$ & $9.99017 \times 10^{-4}$ \\
\hline 0.01 & $45^{\circ}$ & $9.71546 \times 10^{-4}$ & $9.97769 \times 10^{-4}$ & $9.99663 \times 10^{-4}$ & $9.71575 \times 10^{-4}$ & $9.97770 \times 10^{-4}$ & $9.99664 \times 10^{-4}$ \\
\hline 0.01 & $70^{\circ}$ & $9.70462 \times 10^{-4}$ & $9.98777 \times 10^{-4}$ & $1.00073 \times 10^{-3}$ & $9.70484 \times 10^{-4}$ & $9.98780 \times 10^{-4}$ & $1.00073 \times 10^{-3}$ \\
\hline 0.05 & $20^{\circ}$ & $9.68780 \times 10^{-4}$ & $9.93637 \times 10^{-4}$ & $9.95490 \times 10^{-4}$ & $9.68827 \times 10^{-4}$ & $9.93647 \times 10^{-4}$ & $9.95501 \times 10^{-4}$ \\
\hline 0.05 & $45^{\circ}$ & $9.68117 \times 10^{-4}$ & $9.94247 \times 10^{-4}$ & $9.96134 \times 10^{-4}$ & $9.68157 \times 10^{-4}$ & $9.94259 \times 10^{-4}$ & $9.96147 \times 10^{-4}$ \\
\hline 0.05 & $70^{\circ}$ & $9.67039 \times 10^{-4}$ & $9.95254 \times 10^{-4}$ & $9.97196 \times 10^{-4}$ & $9.67072 \times 10^{-4}$ & $9.95268 \times 10^{-4}$ & $9.97210 \times 10^{-4}$ \\
\hline 0.09 & $20^{\circ}$ & $9.60784 \times 10^{-4}$ & $9.85436 \times 10^{-4}$ & $9.87273 \times 10^{-4}$ & $9.60935 \times 10^{-4}$ & $9.85553 \times 10^{-4}$ & $9.87391 \times 10^{-4}$ \\
\hline 0.09 & $45^{\circ}$ & $9.60130 \times 10^{-4}$ & $9.86045 \times 10^{-4}$ & $9.87916 \times 10^{-4}$ & $9.60274 \times 10^{-4}$ & $9.86164 \times 10^{-4}$ & $9.88036 \times 10^{-4}$ \\
\hline 0.09 & $70^{\circ}$ & $9.59067 \times 10^{-4}$ & $9.87050 \times 10^{-4}$ & $9.88975 \times 10^{-4}$ & $9.59204 \times 10^{-4}$ & $9.87171 \times 10^{-4}$ & $9.89097 \times 10^{-4}$ \\
\hline
\end{tabular}

Table 3. Comparison of the orbital frequencies, $\Omega_{r}, \Omega_{\theta}$ and $\Omega_{\phi}$, derived using analytical expressions in this work and numerical integration method in the case of $p=6 M, e=0.7, \theta_{\text {inc }}=20^{\circ}$ and $a=0.9 M$ or $a=M$. Our results are consistent with numerical integration method. Relative errors of the orbital frequencies agree with the accuracy of double precision calculation.

\begin{tabular}{ccccc}
\hline$a / M$ & $\Omega_{r, \theta, \phi}$ & This work & Numerical integration & Absolute value of relative error \\
\hline 0.9 & $\Omega_{r}$ & $1.8928532285101992 \times 10^{-2}$ & $1.8928532285101982 \times 10^{-2}$ & $5.6 \times 10^{-16}$ \\
0.9 & $\Omega_{\theta}$ & $2.7299110395017517 \times 10^{-2}$ & $2.7299110395017506 \times 10^{-2}$ & $4.1 \times 10^{-16}$ \\
0.9 & $\Omega_{\phi}$ & $3.0550463796964692 \times 10^{-2}$ & $3.0550463796964682 \times 10^{-2}$ & $3.6 \times 10^{-16}$ \\
\hline 1 & $\Omega_{r}$ & $1.9343466898960462 \times 10^{-2}$ & $1.9343466898960444 \times 10^{-2}$ & $7.8 \times 10^{-16}$ \\
1 & $\Omega_{\theta}$ & $2.6337035996626332 \times 10^{-2}$ & $2.6337035996626321 \times 10^{-2}$ & $4.3 \times 10^{-16}$ \\
1 & $\Omega_{\phi}$ & $2.9662029663040452 \times 10^{-2}$ & $2.9662029663040452 \times 10^{-2}$ & $4.0 \times 10^{-17}$ \\
\hline
\end{tabular}


the signs of both $\mathrm{d} r / \mathrm{d} \lambda$ and $\mathrm{d} \cos \theta / \mathrm{d} \lambda$ at given $\lambda$-time. In the following subsections, we derive the radial solutions, $r(\lambda), t^{(r)}(\lambda)$ and $\phi^{(r)}(\lambda)$, in section 4.1, and the polar solutions, $\cos \theta(\lambda), t^{(\theta)}(\lambda)$ and $\phi^{(\theta)}(\lambda)$, in section 4.2. Finally, we check the consistency of our analytical results by comparison with the results of earlier literature in section 4.3 .

\subsection{Radial solution : $r(\lambda), t^{(r)}(\lambda)$ and $\phi^{(r)}(\lambda)$}

Solving (12) and (8), we obtain $\lambda(r), t^{(r)}(\lambda)$ and $\phi^{(r)}(\lambda)$ as

$$
\begin{aligned}
\lambda(r) & =\int^{r} \frac{\mathrm{d} r^{\prime}}{\sqrt{R\left(r^{\prime}\right)}}, \\
t^{(r)}(\lambda) & =\int^{r(\lambda)} \frac{T_{r}\left(r^{\prime}\right)-\Upsilon_{t^{(r)}}}{\sqrt{R\left(r^{\prime}\right)}} \mathrm{d} r^{\prime}, \\
\phi^{(r)}(\lambda) & =\int^{r(\lambda)} \frac{\Phi_{r}\left(r^{\prime}\right)-\Upsilon_{\phi^{(r)}}}{\sqrt{R\left(r^{\prime}\right)}} \mathrm{d} r^{\prime} .
\end{aligned}
$$

We derive $r(\lambda)$ inverting $\lambda(r)$. Since the period of $r$-motion with respect to $\lambda$ is $\Lambda_{r}=2 \pi / \Upsilon_{r}$, we map $\lambda$ to $\lambda^{(r)}$ as $\lambda^{(r)}=\lambda-2 \pi\left[\Upsilon_{r} \lambda / 2 \pi\right] / \Upsilon_{r}$, where $[\cdots]$ is the floor function, in the following subsections. In order to investigate the integrations in (22) properly, we have to take account of $r(\lambda=0)$ and the sign of $\mathrm{d} r(\lambda) / \mathrm{d} \lambda$. There exist two cases depending on whether the initial value is $\mathrm{d} r(0) / \mathrm{d} \lambda \geq 0$ or $\mathrm{d} r(0) / \mathrm{d} \lambda \leq 0$. In the following subsections, we consider the two cases separately. We note that the expressions of both $t^{(r)}(\lambda)$ and $\phi^{(r)}(\lambda)$ in the following subsections are valid when $|a| \neq M$. We show $t^{(r)}(\lambda)$ and $\phi^{(r)}(\lambda)$ when $|a|=M$ in Appendix B.

4.1.1. $\mathrm{d} r(0) / \mathrm{d} \lambda \geq 0$ case In this subsection, we consider the case that the initial value of $r(\lambda)$ satisfy $\mathrm{d} r(0) / \mathrm{d} \lambda \geq 0$. We set $r(\lambda=0)=r_{0}^{(1)}$ in this subsection. Then $\lambda(r)$ in (22) can be expressed as

$$
\begin{array}{rlr}
\lambda^{(r)}(r) & =\int_{r_{0}^{(1)}}^{r} \frac{\mathrm{d} r^{\prime}}{\sqrt{R\left(r^{\prime}\right)}}, \\
& =\left[\int_{r_{2}}^{r}-\int_{r_{2}}^{r_{0}^{(1)}}\right] \frac{\mathrm{d} r^{\prime}}{\sqrt{R\left(r^{\prime}\right)}} & r: r_{0}^{(1)} \rightarrow r_{1}, \\
& =\left[-\int_{r_{2}}^{r}+2 \int_{r_{2}}^{r_{1}}-\int_{r_{2}}^{r_{0}^{(1)}}\right] \frac{\mathrm{d} r^{\prime}}{\sqrt{R\left(r^{\prime}\right)}} & r: r_{1} \rightarrow r_{2}, \\
& =\left[\int_{r_{2}}^{r}+2 \int_{r_{2}}^{r_{1}}-\int_{r_{2}}^{r_{0}^{(1)}}\right] \frac{\mathrm{d} r^{\prime}}{\sqrt{R\left(r^{\prime}\right)}} & r: r_{2} \rightarrow r_{0}^{(1)} .
\end{array}
$$

Thus we find the solution as

$$
\lambda^{(r)}(r)= \begin{cases}\lambda_{0}^{(r)}(r)-\Lambda_{r}^{(1)} & r: r_{0}^{(1)} \rightarrow r_{1}, \\ -\lambda_{0}^{(r)}(r)+\Lambda_{r}-\Lambda_{r}^{(1)} & r: r_{1} \rightarrow r_{2}, \\ \lambda_{0}^{(r)}(r)+\Lambda_{r}-\Lambda_{r}^{(1)} & r: r_{2} \rightarrow r_{0}^{(1)}\end{cases}
$$


Analytical solutions of bound timelike geodesic orbits in Kerr spacetime

where

$$
\lambda_{0}^{(r)}(r)=\frac{1}{\sqrt{1-\mathcal{E}^{2}}} \frac{2}{\sqrt{\left(r_{1}-r_{3}\right)\left(r_{2}-r_{4}\right)}} F\left(\arcsin y_{r}, k_{r}\right)
$$

and $\Lambda_{r}^{(1)}=\lambda_{0}^{(r)}\left(r_{0}^{(1)}\right)$.

Inverting (24), we derive $r(\lambda)$ as

$$
r(\lambda)=\frac{r_{3}\left(r_{1}-r_{2}\right) \operatorname{sn}^{2}\left(u_{r}(\lambda), k_{r}\right)-r_{2}\left(r_{1}-r_{3}\right)}{\left(r_{1}-r_{2}\right) \operatorname{sn}^{2}\left(u_{r}(\lambda), k_{r}\right)-\left(r_{1}-r_{3}\right)}
$$

where $\operatorname{sn}(u, k)$ is Jacobi's elliptic function which is defined as the inverse function of the incomplete elliptic integrals, $u=F(\varphi, k)$, and

$$
u_{r}(\lambda)= \begin{cases}2 K\left(k_{r}\right)\left(\lambda^{(r)}+\Lambda_{r}^{(1)}\right) / \Lambda_{r} & \left(0 \leq \lambda^{(r)} \leq \Lambda_{r} / 2-\Lambda_{r}^{(1)}\right) \\ 2 K\left(k_{r}\right)\left(-\lambda^{(r)}+\Lambda_{r}-\Lambda_{r}^{(1)}\right) / \Lambda_{r} & \left(\Lambda_{r} / 2-\Lambda_{r}^{(1)} \leq \lambda^{(r)} \leq \Lambda_{r}-\Lambda_{r}^{(1)}\right) \\ 2 K\left(k_{r}\right)\left(\lambda^{(r)}-\Lambda_{r}+\Lambda_{r}^{(1)}\right) / \Lambda_{r} & \left(\Lambda_{r}-\Lambda_{r}^{(1)} \leq \lambda^{(r)} \leq \Lambda_{r}\right)\end{cases}
$$

Combining the results of (23) with the results of Appendix A, we can derive $t^{(r)}$ and $\phi^{(r)}$ in (22) as

$$
\begin{aligned}
& t^{(r)}=\frac{2}{\sqrt{\left(1-\mathcal{E}^{2}\right)\left(r_{1}-r_{3}\right)\left(r_{2}-r_{4}\right)}} \\
& \quad \times\left\{\frac { \mathcal { E } } { 2 } \left[\left(r_{2}-r_{3}\right)\left(r_{1}+r_{2}+r_{3}+r_{4}\right) \tilde{\Pi}_{r}\left(\psi_{r},-h_{r}, k_{r}\right)\right.\right. \\
&\left.+\left(r_{1}-r_{3}\right)\left(r_{2}-r_{4}\right) \tilde{E}_{r}\left(\psi_{r}, h_{r}, k_{r}\right)\right] \\
&+ 2 M \mathcal{E}\left(r_{2}-r_{3}\right) \tilde{\Pi}_{r}\left(\psi_{r},-h_{r}, k_{r}\right) \\
&-\frac{2 M}{r_{+}-r_{-}}\left[\frac{\left(4 M^{2} \mathcal{E}-a \mathcal{L}_{z}\right) r_{+}-2 M a^{2} \mathcal{E}}{r_{3}-r_{+}} \frac{r_{2}-r_{3}}{r_{2}-r_{+}} \tilde{\Pi}_{r}\left(\psi_{r},-h_{+}, k_{r}\right)\right. \\
&-(+\leftrightarrow-)]\}, \\
& \phi^{(r)}=-\frac{2 a}{\left(r_{+}-\right.}\left.r_{-}\right) \sqrt{\left(1-\mathcal{E}^{2}\right)\left(r_{1}-r_{3}\right)\left(r_{2}-r_{4}\right)}\left[\frac{(28)}{\left(r_{3}-r_{+}\right)\left(r_{2}-r_{+}\right)}\right) \\
&-(+\leftrightarrow-)],(28)
\end{aligned}
$$

where $\psi_{r}=\arcsin \left[\operatorname{sn}\left(u_{r}, k_{r}\right)\right], \tilde{E}_{r}\left(\psi_{r}, c, k_{r}\right)=E_{r}\left(\psi_{r}, c, k_{r}\right)-\frac{\Upsilon_{r} \lambda^{(r)}}{\pi} E\left(k_{r}\right), \tilde{\Pi}_{r}\left(\psi_{r}, c, k_{r}\right)=$ $\Pi_{r}\left(\psi_{r}, c, k_{r}\right)-\frac{\Upsilon_{r} \lambda^{(r)}}{\pi} \Pi\left(c, k_{r}\right)$ and

$$
\begin{aligned}
E_{r}^{(0)}\left(\psi_{r}, c, k_{r}\right) & =E\left(\psi_{r}, k_{r}\right)+\frac{\sin \psi_{r} \sqrt{\left(1-\sin ^{2} \psi_{r}\right)\left(1-k_{r}^{2} \sin ^{2} \psi_{r}\right)}}{\sin ^{2} \psi_{r}-c^{-1}}, \\
E_{r}\left(\psi_{r}, c, k_{r}\right) & =E_{r}^{(0)}\left(\psi_{r}, c, k_{r}\right)-E_{r}^{(0)}\left(\psi_{r}(0), c, k_{r}\right) \\
& \quad \text { for } \quad 0 \leq \lambda^{(r)} \leq \Lambda_{r} / 2-\Lambda_{r}^{(1)}, \\
& =E_{r}^{(0)}\left(\psi_{r}, c, k_{r}\right)+2 E\left(k_{r}\right)-E_{r}^{(0)}\left(\psi_{r}(0), c, k_{r}\right) \\
& \text { for } \quad \Lambda_{r} / 2-\Lambda_{r}^{(1)} \leq \lambda^{(r)} \leq \Lambda_{r}-\Lambda_{r}^{(1)}, \\
& \quad \text { for }\left(k_{r}\right)-E_{r}^{(0)}\left(\psi_{r}(0), c, k_{r}\right) \\
\prod_{r}\left(\psi_{r}, c, \Lambda_{r}^{(1)} \leq \lambda^{(r)} \leq \Lambda_{r},\right. & \\
& \Pi\left(\psi_{r}, c, k_{r}\right)-\Pi\left(\psi_{r}(0), c, k_{r}\right) \quad \text { for } \quad 0 \leq \lambda^{(r)} \leq \Lambda_{r} / 2-\Lambda_{r}^{(1)}, \\
& =-\Pi\left(\psi_{r}, c, k_{r}\right)+2 \Pi\left(c, k_{r}\right)-\Pi\left(\psi_{r}(0), c, k_{r}\right)
\end{aligned}
$$


Analytical solutions of bound timelike geodesic orbits in Kerr spacetime

$$
\begin{array}{r}
\text { for } \quad \Lambda_{r} / 2-\Lambda_{r}^{(1)} \leq \lambda^{(r)} \leq \Lambda_{r}-\Lambda_{r}^{(1)}, \\
=\Pi\left(\psi_{r}, c, k_{r}\right)+2 \Pi\left(c, k_{r}\right)-\Pi\left(\psi_{r}(0), c, k_{r}\right) \\
\text { for } \quad \Lambda_{r}-\Lambda_{r}^{(1)} \leq \lambda^{(r)} \leq \Lambda_{r} .
\end{array}
$$

4.1.2. $\mathrm{d} r(0) / \mathrm{d} \lambda \leq 0$ case In this subsection, we consider the case that the initial value of $r(\lambda)$ satisfy $\mathrm{d} r(0) / \mathrm{d} \lambda \leq 0$. We set $r(\lambda=0)=r_{0}^{(2)}$ in this subsection. Then $\lambda(r)$ in (22) can be expressed as

$$
\begin{array}{rlr}
\lambda^{(r)}(r) & =\int_{r_{0}^{(2)}}^{r} \frac{\mathrm{d} r^{\prime}}{\sqrt{R\left(r^{\prime}\right)}}, \\
& =\left[-\int_{r_{2}}^{r}+\int_{r_{2}}^{r_{0}^{(2)}}\right] \frac{\mathrm{d} r^{\prime}}{\sqrt{R\left(r^{\prime}\right)}} & r: r_{0}^{(2)} \rightarrow r_{2}, \\
& =\left[\int_{r_{2}}^{r}+\int_{r_{2}}^{r_{0}^{(2)}}\right] \frac{\mathrm{d} r^{\prime}}{\sqrt{R\left(r^{\prime}\right)}} & \\
& =\left[-\int_{r_{2}}^{r}+2 \int_{r_{2}}^{r_{1}}+\int_{r_{2}}^{r_{0}^{(2)}}\right] \frac{\mathrm{d} r^{\prime}}{\sqrt{R\left(r^{\prime}\right)}} & \\
& r: r_{1} \rightarrow r_{1},
\end{array}
$$

Thus we find the solution as

$$
\lambda^{(r)}(r)= \begin{cases}-\lambda_{0}^{(r)}(r)+\Lambda_{r}^{(2)} & r: r_{0}^{(2)} \rightarrow r_{2}, \\ \lambda_{0}^{(r)}(r)+\Lambda_{r}^{(2)} & r: r_{2} \rightarrow r_{1}, \\ -\lambda_{0}^{(r)}(r)+\Lambda_{r}+\Lambda_{r}^{(2)} & r: r_{1} \rightarrow r_{0}^{(2)},\end{cases}
$$

where $\Lambda_{r}^{(2)}=\lambda_{0}^{(r)}\left(r_{0}^{(2)}\right)$.

Then we obtain $r(\lambda)$ in the same form in (26) inverting (31), but $u_{r}(\lambda)$ in (26) is modified as

$$
u_{r}(\lambda)= \begin{cases}2 K\left(k_{r}\right)\left(-\lambda^{(r)}+\Lambda_{r}^{(2)}\right) / \Lambda_{r} & \left(0 \leq \lambda^{(r)} \leq \Lambda_{r}^{(2)}\right), \\ 2 K\left(k_{r}\right)\left(\lambda^{(r)}-\Lambda_{r}^{(2)}\right) / \Lambda_{r} & \left(\Lambda_{r}^{(2)} \leq \lambda^{(r)} \leq \Lambda_{r} / 2+\Lambda_{r}^{(2)}\right), \\ 2 K\left(k_{r}\right)\left(-\lambda^{(r)}+\Lambda_{r}+\Lambda_{r}^{(2)}\right) / \Lambda_{r} & \left(\Lambda_{r} / 2+\Lambda_{r}^{(2)} \leq \lambda^{(r)} \leq \Lambda_{r}\right) .\end{cases}
$$

Combining the results of (30) with the results of Appendix A, we can derive $t^{(r)}$ and $\phi^{(r)}$ in the same form in (28), but $E_{r}\left(\psi_{r}, c, k_{r}\right)$ and $\Pi_{r}\left(\psi_{r}, c, k_{r}\right)$ are modified as

$$
\begin{gathered}
E_{r}\left(\psi_{r}, c, k_{r}\right)=-E_{r}^{(0)}\left(\psi_{r}, c, k_{r}\right)+E_{r}^{(0)}\left(\psi_{r}(0), c, k_{r}\right) \\
\quad \text { for } 0 \leq \lambda^{(r)} \leq \Lambda_{r}^{(2)}, \\
=E_{r}^{(0)}\left(\psi_{r}, c, k_{r}\right)+E_{r}^{(0)}\left(\psi_{r}(0), c, k_{r}\right) \\
\quad \text { for } \quad \Lambda_{r}^{(2)} \leq \lambda^{(r)} \leq \Lambda_{r} / 2+\Lambda_{r}^{(2)}, \\
=-E_{r}^{(0)}\left(\psi_{r}, c, k_{r}\right)+\underset{r}{2 E\left(k_{r}\right)+E_{r}^{(0)}\left(\psi_{r}(0), c, k_{r}\right)} \\
\quad \text { for } \Lambda_{r} / 2+\Lambda_{r}^{(2)} \leq \lambda^{(r)} \leq \Lambda_{r}, \\
\Pi_{r}\left(\psi_{r}, c, k_{r}\right)=-\Pi\left(\psi_{r}, c, k_{r}\right)+\prod\left(\psi_{r}(0), c, k_{r}\right) \\
\quad \text { for } 0 \leq \lambda^{(r)} \leq \Lambda_{r}^{(2)}, \\
=\prod\left(\psi_{r}, c, k_{r}\right)+\Pi\left(\psi_{r}(0), c, k_{r}\right) \\
\quad \text { for } \quad \Lambda_{r}^{(2)} \leq \lambda^{(r)} \leq \Lambda_{r} / 2+\Lambda_{r}^{(2)}, \\
=-\Pi\left(\psi_{r}, c, k_{r}\right)+2 \Pi\left(c, k_{r}\right)+\Pi\left(\psi_{r}(0), c, k_{r}\right) \\
\quad \text { for } \quad \Lambda_{r} / 2+\Lambda_{r}^{(2)} \leq \lambda^{(r)} \leq \Lambda_{r} .
\end{gathered}
$$




\subsection{Polar Solution: $\cos \theta(\lambda), t^{(\theta)}(\lambda)$ and $\phi^{(\theta)}(\lambda)$}

Solving (12) and (8) , we obtain $\lambda(\cos \theta), t^{(\theta)}(\lambda)$ and $\phi^{(\theta)}(\lambda)$ as

$$
\begin{aligned}
\lambda(\theta) & =\int^{\cos \theta} \frac{\mathrm{d} \cos \theta^{\prime}}{\sqrt{\Theta\left(\cos \theta^{\prime}\right)}}, \\
t^{(\theta)}(\lambda) & =\int^{\cos \theta} \frac{T_{\theta}\left(\cos \theta^{\prime}\right)-\Upsilon_{t^{(\theta)}}}{\sqrt{\Theta\left(\cos \theta^{\prime}\right)}} \mathrm{d} \cos \theta^{\prime}, \\
\phi^{(\theta)}(\lambda) & =\int^{\cos \theta} \frac{\Phi_{\theta}\left(\cos \theta^{\prime}\right)-\Upsilon_{\phi^{(\theta)}}}{\sqrt{\Theta\left(\cos \theta^{\prime}\right)}} \mathrm{d} \cos \theta^{\prime} .
\end{aligned}
$$

We derive $\cos \theta(\lambda)$ inverting $\lambda(\theta)$. Since the period of $\theta$-motion with respect to $\lambda$ is $\Lambda_{\theta}=2 \pi / \Upsilon_{\theta}$, we map $\lambda$ to $\lambda^{(\theta)}$ as $\lambda^{(\theta)}=\lambda-2 \pi\left[\Upsilon_{\theta} \lambda / 2 \pi\right] / \Upsilon_{\theta}$ in the following subsections. In order to investigate the integrations in (34) properly, we have to take account of $\cos \theta(\lambda=0)$ and the sign of $\mathrm{d} \cos \theta(\lambda) / \mathrm{d} \lambda$. There exist two cases depending on whether the initial value is $d \cos \theta(0) / d \lambda \geq 0$ or $d \cos \theta(0) / d \lambda \leq 0$. In the following subsections, we consider the two cases separately.

4.2.1. $d \cos \theta(0) / d \lambda \geq 0$ case In this subsection, we consider the case that the initial value of $\cos \theta(\lambda)$ satisfies $\mathrm{d} \cos \theta(0) / \mathrm{d} \lambda \geq 0$. We set $\theta(\lambda=0)=\theta_{0}^{(1)}$ in this subsection. Then $\lambda(\theta)$ in (34) can be expressed as

$$
\begin{aligned}
& \lambda^{(\theta)}(\theta) \quad=\int_{\cos \theta_{0}^{(1)}}^{\cos \theta} \frac{\mathrm{d} \cos \theta^{\prime}}{\sqrt{\Theta\left(\cos \theta^{\prime}\right)}}, \\
& =\left[\int_{0}^{\cos \theta}-\int_{0}^{\cos \theta_{0}^{(1)}}\right] \frac{\mathrm{d} \cos \theta^{\prime}}{\sqrt{\Theta\left(\cos \theta^{\prime}\right)}} \quad \theta: \theta_{0}^{(1)} \rightarrow \theta_{\min }, \\
& =\left[-\int_{0}^{\cos \theta}+2 \int_{0}^{\cos \theta_{\min }}-\int_{0}^{\cos \theta_{0}^{(1)}}\right] \frac{\mathrm{d} \cos \theta^{\prime}}{\sqrt{\Theta\left(\cos \theta^{\prime}\right)}} \quad \theta: \theta_{\min } \rightarrow \pi-\theta_{\min }, \\
& =\left[\int_{0}^{\cos \theta}+4 \int_{0}^{\cos \theta_{\min }}-\int_{0}^{\cos \theta_{0}^{(1)}}\right] \frac{\mathrm{d} \cos \theta^{\prime}}{\sqrt{\Theta\left(\cos \theta^{\prime}\right)}} \quad \theta: \pi-\theta_{\min } \rightarrow \theta_{0}^{(1)}(.35)
\end{aligned}
$$

Thus we find the solution as

$$
\lambda^{(\theta)}(\theta)= \begin{cases}\lambda_{0}^{(\theta)}(\theta)-\Lambda_{\theta}^{(1)} & \theta: \theta_{0}^{(1)} \rightarrow \theta_{\min }, \\ -\lambda_{0}^{(\theta)}(\theta)+\Lambda_{\theta} / 2-\Lambda_{\theta}^{(1)} & \theta: \theta_{\min } \rightarrow \pi-\theta_{\min }, \\ \lambda_{0}^{(\theta)}(\theta)+\Lambda_{\theta}-\Lambda_{\theta}^{(1)} & \theta: \pi-\theta_{\min } \rightarrow \theta_{0}^{(1)},\end{cases}
$$

where

$$
\lambda_{0}^{(\theta)}(\theta)=\frac{1}{\mathcal{L}_{z} \sqrt{\epsilon_{0} z_{+}}} F\left(\arcsin y_{\theta}, k_{\theta}\right)
$$

and $\Lambda_{\theta}^{(1)}=\lambda_{0}^{(\theta)}\left(\theta_{0}^{(1)}\right)$.

Inverting (36) , we derive $\cos \theta(\lambda)$ as

$$
\cos \theta(\lambda)=\sqrt{z_{-}} \operatorname{sn}\left(u_{\theta}(\lambda), k_{\theta}\right),
$$


Analytical solutions of bound timelike geodesic orbits in Kerr spacetime

where

$$
u_{\theta}(\lambda)= \begin{cases}4 K\left(k_{\theta}\right)\left(\lambda^{(\theta)}+\Lambda_{\theta}^{(1)}\right) / \Lambda_{\theta} & \left(0 \leq \lambda^{(\theta)} \leq \Lambda_{\theta} / 4-\Lambda_{\theta}^{(1)}\right), \\ 4 K\left(k_{\theta}\right)\left(-\lambda^{(\theta)}+\Lambda_{\theta} / 2-\Lambda_{\theta}^{(1)}\right) / \Lambda_{\theta} & \left(\Lambda_{\theta} / 4-\Lambda_{\theta}^{(1)} \leq \lambda^{(\theta)} \leq 3 \Lambda_{\theta} / 4-\Lambda_{\theta}^{(1)}\right), \\ 4 K\left(k_{\theta}\right)\left(\lambda^{(\theta)}-\Lambda_{\theta}+\Lambda_{\theta}^{(1)}\right) / \Lambda_{\theta} & \left(3 \Lambda_{\theta} / 4-\Lambda_{\theta}^{(1)} \leq \lambda^{(\theta)} \leq \Lambda_{\theta}\right) .\end{cases}
$$

Using the results of (35), we derive $t^{(\theta)}$ and $\phi^{(\theta)}$ as

$$
\begin{aligned}
t^{(\theta)} & =\frac{a^{2} \mathcal{E} z_{+}}{\mathcal{L}_{z} \sqrt{\epsilon_{0} z_{+}}}\left[\frac{2 \Upsilon_{\theta} \lambda^{(\theta)}}{\pi} E\left(k_{\theta}\right)-E_{\theta}\left(\psi_{\theta}, k_{\theta}\right)\right], \\
\phi^{(\theta)} & =\frac{1}{\sqrt{\epsilon_{0} z_{+}}}\left[\Pi_{\theta}\left(\psi_{\theta},-z_{-}, k_{\theta}\right)-\frac{2 \Upsilon_{\theta} \lambda^{(\theta)}}{\pi} \Pi\left(-z_{-}, k_{\theta}\right)\right],
\end{aligned}
$$

where $\psi_{\theta}=\arcsin \left[\operatorname{sn}\left(u_{\theta}, k_{\theta}\right)\right]$ and

$$
\begin{aligned}
& E_{\theta}\left(\psi_{\theta}, k_{\theta}\right) \quad=E\left(\psi_{\theta}, k_{\theta}\right)-E\left(\psi_{\theta}(0), k_{\theta}\right) \\
& \text { for } \quad 0 \leq \lambda^{(\theta)} \leq \Lambda_{\theta} / 4-\Lambda_{\theta}^{(1)} \text {, } \\
& =-E\left(\psi_{\theta}, k_{\theta}\right)+2 E\left(k_{\theta}\right)-E\left(\psi_{\theta}(0), k_{\theta}\right) \\
& \text { for } \quad \Lambda_{\theta} / 4-\Lambda_{\theta}^{(1)} \leq \lambda^{(\theta)} \leq 3 \Lambda_{\theta} / 4-\Lambda_{\theta}^{(1)} \text {, } \\
& =E\left(\psi_{\theta}, k_{\theta}\right)+4 E\left(k_{\theta}\right)-E\left(\psi_{\theta}(0), k_{\theta}\right) \\
& \text { for } \quad 3 \Lambda_{\theta} / 4-\Lambda_{\theta}^{(1)} \leq \lambda^{(\theta)} \leq \Lambda_{\theta} \text {, } \\
& \Pi_{\theta}\left(\psi_{\theta}, c, k_{\theta}\right)=\Pi\left(\psi_{\theta}, c, k_{\theta}\right)-\Pi\left(\psi_{\theta}(0), c, k_{\theta}\right) \\
& \text { for } \quad 0 \leq \lambda^{(\theta)} \leq \Lambda_{\theta} / 4-\Lambda_{\theta}^{(1)}, \\
& =-\Pi\left(\psi_{\theta}, c, k_{\theta}\right)+2 \Pi\left(c, k_{\theta}\right)-\Pi\left(\psi_{\theta}(0), c, k_{\theta}\right) \\
& \text { for } \quad \Lambda_{\theta} / 4-\Lambda_{\theta}^{(1)} \leq \lambda^{(\theta)} \leq 3 \Lambda_{\theta} / 4-\Lambda_{\theta}^{(1)}, \\
& =\Pi\left(\psi_{\theta}, c, k_{\theta}\right)+4 \Pi\left(c, k_{\theta}\right)-\Pi\left(\psi_{\theta}(0), c, k_{\theta}\right) \\
& \text { for } \quad 3 \Lambda_{\theta} / 4-\Lambda_{\theta}^{(1)} \leq \lambda^{(\theta)} \leq \Lambda_{\theta} \text {. }
\end{aligned}
$$

4.2.2. $\mathrm{d} \cos \theta(0) / \mathrm{d} \lambda \leq 0$ case In this subsection, we consider the case that the initial value of $\cos \theta(\lambda)$ satisfies $\mathrm{d} \cos \theta(0) / \mathrm{d} \lambda \leq 0$. We set $\theta(\lambda=0)=\theta_{0}^{(2)}$ in this subsection. Then $\lambda(\theta)$ in (34) can be expressed as

$$
\begin{aligned}
& \lambda^{(\theta)}(\theta) \quad=\int_{\cos \theta_{0}^{(2)}}^{\cos \theta} \frac{\mathrm{d} \cos \theta^{\prime}}{\sqrt{\Theta\left(\cos \theta^{\prime}\right)}}, \\
& =\left[-\int_{0}^{\cos \theta}+\int_{0}^{\cos \theta_{0}^{(2)}}\right] \frac{\mathrm{d} \cos \theta^{\prime}}{\sqrt{\Theta\left(\cos \theta^{\prime}\right)}} \quad \theta: \theta_{0}^{(2)} \rightarrow \pi-\theta_{\min }, \\
& =\left[\int_{0}^{\cos \theta}+2 \int_{0}^{\cos \theta_{\min }}+\int_{0}^{\cos \theta_{0}^{(2)}}\right] \frac{\mathrm{d} \cos \theta^{\prime}}{\sqrt{\Theta\left(\cos \theta^{\prime}\right)}} \quad \theta: \pi-\theta_{\min } \rightarrow \theta_{\min }, \\
& =\left[-\int_{0}^{\cos \theta}+4 \int_{0}^{\cos \theta_{\min }}+\int_{0}^{\cos \theta_{0}^{(2)}}\right] \frac{\mathrm{d} \cos \theta^{\prime}}{\sqrt{\Theta\left(\cos \theta^{\prime}\right)}} \quad \theta: \theta_{\min } \rightarrow \theta_{0}^{(2)} .
\end{aligned}
$$

Thus we find the solution as

$$
\lambda^{(\theta)}(\theta)= \begin{cases}-\lambda_{0}^{(\theta)}(\theta)+\Lambda_{\theta}^{(2)} & \theta: \theta_{0}^{(2)} \rightarrow \pi-\theta_{\min }, \\ \lambda_{0}^{(\theta)}(\theta)+\Lambda_{\theta} / 2+\Lambda_{\theta}^{(2)} & \theta: \pi-\theta_{\min } \rightarrow \theta_{\min }, \\ -\lambda_{0}^{(\theta)}(\theta)+\Lambda_{\theta}+\Lambda_{\theta}^{(2)} & \theta: \theta_{\min } \rightarrow \theta_{0}^{(2)}\end{cases}
$$


where $\Lambda_{\theta}^{(2)}=\lambda_{0}^{(\theta)}\left(\theta_{0}^{(2)}\right)$.

Then we obtain $\cos \theta(\lambda)$ in the same form in (38) inverting (43), but $u_{\theta}(\lambda)$ in (38) is modified as

$$
u_{\theta}(\lambda)= \begin{cases}4 K\left(k_{\theta}\right)\left(-\lambda^{(\theta)}+\Lambda_{\theta}^{(2)}\right) / \Lambda_{\theta} & \left(0 \leq \lambda^{(\theta)} \leq \Lambda_{\theta} / 4+\Lambda_{\theta}^{(2)}\right), \\ 4 K\left(k_{\theta}\right)\left(\lambda^{(\theta)}-\Lambda_{\theta} / 2-\Lambda_{\theta}^{(2)}\right) / \Lambda_{\theta} & \left(\Lambda_{\theta} / 4+\Lambda_{\theta}^{(2)} \leq \lambda^{(\theta)} \leq 3 \Lambda_{\theta} / 4+\Lambda_{\theta}^{(2)}\right), \\ 4 K\left(k_{\theta}\right)\left(-\lambda^{(\theta)}+\Lambda_{\theta}+\Lambda_{\theta}^{(2)}\right) / \Lambda_{\theta} & \left(3 \Lambda_{\theta} / 4+\Lambda_{\theta}^{(2)} \leq \lambda^{(\theta)} \leq \Lambda_{\theta}\right) .\end{cases}
$$

Using the results of (42), we derive $t^{(\theta)}$ and $\phi^{(\theta)}$ as in the same form in (39), but $E_{\theta}\left(\psi_{\theta}, k_{\theta}\right)$ and $\Pi_{\theta}\left(\psi_{\theta}, c, k_{\theta}\right)$ are modified as

$$
\begin{aligned}
& E_{\theta}\left(\psi_{\theta}, k_{\theta}\right) \quad=-E\left(\psi_{\theta}, k_{\theta}\right)+E\left(\psi_{\theta}(0), k_{\theta}\right) \\
& \text { for } \quad 0 \leq \lambda^{(\theta)} \leq \Lambda_{\theta} / 4+\Lambda_{\theta}^{(2)} \text {, } \\
& =E\left(\psi_{\theta}, k_{\theta}\right)+2 E\left(k_{\theta}\right)+E\left(\psi_{\theta}(0), k_{\theta}\right) \\
& \text { for } \quad \Lambda_{\theta} / 4+\Lambda_{\theta}^{(2)} \leq \lambda^{(\theta)} \leq 3 \Lambda_{\theta} / 4+\Lambda_{\theta}^{(2)} \text {, } \\
& =-E\left(\psi_{\theta}, k_{\theta}\right)+4 E\left(k_{\theta}\right)+E\left(\psi_{\theta}(0), k_{\theta}\right) \\
& \text { for } 3 \Lambda_{\theta} / 4+\Lambda_{\theta}^{(2)} \leq \lambda^{(\theta)} \leq \Lambda_{\theta} \text {, } \\
& \Pi_{\theta}\left(\psi_{\theta}, c, k_{\theta}\right)=-\Pi\left(\psi_{\theta}, c, k_{\theta}\right)+\Pi\left(\psi_{\theta}(0), c, k_{\theta}\right) \\
& \text { for } \quad 0 \leq \lambda^{(\theta)} \leq \Lambda_{\theta} / 4+\Lambda_{\theta}^{(2)} \text {, } \\
& =\Pi\left(\psi_{\theta}, c, k_{\theta}\right)+2 \Pi\left(c, k_{\theta}\right)+\Pi\left(\psi_{\theta}(0), c, k_{\theta}\right) \\
& \text { for } \quad \Lambda_{\theta} / 4+\Lambda_{\theta}^{(2)} \leq \lambda^{(\theta)} \leq 3 \Lambda_{\theta} / 4+\Lambda_{\theta}^{(2)}, \\
& =-\Pi\left(\psi_{\theta}, c, k_{\theta}\right)+4 \Pi\left(c, k_{\theta}\right)+\Pi\left(\psi_{\theta}(0), c, k_{\theta}\right) \\
& \text { for } 3 \Lambda_{\theta} / 4+\Lambda_{\theta}^{(2)} \leq \lambda^{(\theta)} \leq \Lambda_{\theta} \text {. }
\end{aligned}
$$

\subsection{Consistency check of the analytical solution}

In this subsection, we compare the analytical results of bound geodesics, $t(\lambda), r(\lambda)$, $\cos \theta(\lambda)$ and $\phi(\lambda)$, with earlier literature as a consistency check. We can compare $r(\lambda)$ in (26) with that in [13, 23], in which the integral of motion, $\int \mathrm{d} r / \sqrt{R(r)}=$ $\int \mathrm{d} \cos \theta / \sqrt{\Theta(\cos \theta)}$, is solved in terms of Jacobi's elliptic function using $\theta$ as the independent variable. Since [13] deals with null geodesics and [23] does not give explicit expressions including the turning points, we can not compare them exactly. However, we find that the formal expressions of $r(\lambda)$ in this paper and [13, 23] are consistent. We can also compare the formal expression of $\cos \theta(\lambda)$ in (38) with that in [13], in which the integral of motion is solved in terms of Jacobi's elliptic function using $r$ as the independent variable. Then we find that the formal expressions of $\cos \theta(\lambda)$ in this paper and [13] are consistent. Although [13] derived $t$ and $\phi$ in terms of Carlson elliptic integrals [15], it seems difficult to compare the expressions of both $t$ and $\phi$ in this paper with that of null geodesics in 13. Thus we compare the expressions of each integrals such as $\int_{r_{2}}^{r}\left(r^{\prime}\right)^{2} \mathrm{~d} r^{\prime} / \sqrt{R\left(r^{\prime}\right)}$ in Appendix A with that in [15], in which formulas for $\int_{y}^{x} \prod_{i=1}^{5}\left(a_{i}+b_{i} t\right)^{p_{i} / 2} \mathrm{~d} t$ are derived in terms of Carlson elliptic integrals, where all quantities are real, $x>y$ and $a_{i}+b_{i} t>0$ for $y<t<x$. Using transformation of Carlson elliptic integrals of the third kind [24], it is straightforward to check $\int_{r_{2}}^{r}\left(r^{\prime}\right)^{2} \mathrm{~d} r^{\prime} / \sqrt{R\left(r^{\prime}\right)}$ in Appendix A agrees with the corresponding formula in [15]. We can also check the 
other integrals in this paper agree with that in [15]. These analytical checks show that the expressions in this paper are consistent.

Moreover, we compare the analytical results in this paper with that of numerical integration method. It is a good check on the results that both the coefficients and turning points in each integrals are consistent. In numerical integration of geodesic equation, as explained in section 2, we transform $r$ as $r=p M /(1+e \cos \psi)$ and $\cos \theta$ as $\cos \theta=\cos \theta_{\min } \cos \chi$ respectively. Using (2), we obtain the following set of differential equations [9]

$$
\begin{aligned}
& \frac{\mathrm{d} \psi}{\mathrm{d} \lambda}=\frac{M \sqrt{\left(1-\mathcal{E}^{2}\right)\left\{\left(p-p_{3}\right)-e\left(p+p_{3} \cos \psi\right)\right\}\left\{\left(p-p_{4}\right)+e\left(p-p_{4} \cos \psi\right)\right\}}}{1-e^{2}}, \\
& \frac{\mathrm{d} \chi}{\mathrm{d} \lambda}=\sqrt{a^{2}\left(1-\mathcal{E}^{2}\right)\left(z_{+}-z_{-} \cos ^{2} \chi\right)}, \\
& \frac{\mathrm{d} t}{\mathrm{~d} \lambda}=T_{\mathrm{r}}(r)+T_{\theta}(\cos \theta)+a \mathcal{L}_{z}, \\
& \frac{\mathrm{d} \phi}{\mathrm{d} \lambda}=\Phi_{\mathrm{r}}(r)+\Phi_{\theta}(\cos \theta)-a \mathcal{E},
\end{aligned}
$$

where $p_{3}=r_{3}(1-e) / M$ and $p_{4}=r_{4}(1+e) / M$. We can numerically solve (46) accurately without taking account of the turning points of both $r$ and $\cos \theta$ because both $\psi$ and $\chi$ are monotonic increasing functions of time.

In figure1, we compare the results of our analytical expressions with the results from the numerical integration method. We choose orbital parameters as $a=0.9 M, p=4 M$, $e=0.7$ and $\theta_{\mathrm{inc}}=40^{\circ}$. And set the initial values of $\psi$ and $\chi$ as $\psi(0)=0$ and $\chi(0)=0$ respectively, which correspond to $r(0)=r_{0}^{(1)}=p M /(1+e)$ and $\theta(0)=\theta_{0}^{(1)}=\pi / 2$. For numerical integration of (46), we use the 4th order Runge-Kutta method with nonadaptive step-size control [17]. This figure shows that the analytical solutions of geodesic equation in this paper exactly represent the solutions of bound geodesic orbits around a Kerr black hole.

\section{Summary}

We have derived analytical solutions for bound timelike geodesics in Kerr spacetime. This is the first time that analytical expressions of the fundamental frequencies are derived in terms of the elliptic integrals. The analytical expressions of the orbits, $(t, r, \cos \theta, \phi)$, have been also derived in terms of the elliptic integrals using Mino time as the independent variable for the first time. Since Mino time decouples the $r$ and $\theta$ motion, it leads to forms simpler than that in [13] for null case if we suitably transform variables, $r$ and $\theta$. We checked the consistency of the analytical expressions comparing them with the analytical expressions for the other cases, post-Newtonian approximation and numerical integration method.

We can apply these solutions to the computation of gravitational waves from EMRIs. Gravitational waves from EMRIs are described by the Teukolsky formalism [25]. In the frequency domain calculation of the Teukolsky formalism [26, 27], we can use

the analytical solutions directory [28, 29] and compute the orbits more accurately than 


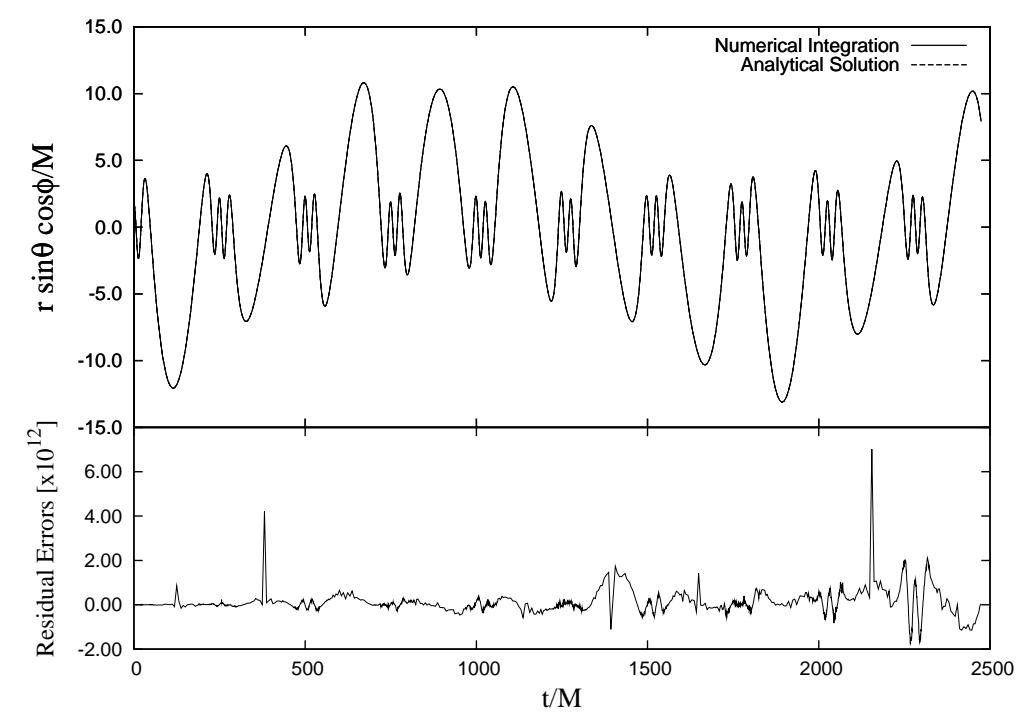

Figure 1. Comparison of the function $x(t)=r(t) \sin \theta(t) \cos \phi(t)$ computed using the analytical expressions of this paper with the result of a numerical integration. In this figure, we set orbital elements as $a=0.9 M, p=4 M, e=0.7$ and $\theta_{\text {inc }}=40^{\circ}$. And we set initial value of $\psi$ and $\chi$ as $\psi(0)=0$ and $\chi(0)=0$ respectively, which correspond to $r(0)=r_{0}^{(1)}=p M /(1+e)$ and $\theta(0)=\theta_{0}^{(1)}=\pi / 2$. Upper figure shows plots of both analytical solution, $x_{\mathrm{A}}(t)$, and numerical integration method, $x_{\mathrm{N}}(t)$. Lower figure shows the residual errors between the results of both analytical solution and numerical integration method, $x_{\mathrm{A}}(t)-x_{\mathrm{N}}(t)$.

numerical integration of geodesic equation. In principle, we can compute the orbits with machine accuracy. Using the analytical expressions of radial and polar motion in this paper, we showed that the analytical expressions enable one to compute gravitational waves from EMRIs very accurately [29]. Although it may takes longer time to compute the orbits using the analytical solutions than using numerical integration method [12], but see [13], it is not serious in computing gravitational waves. This is because we compute orbits only for one orbital period of radial and polar motion, $\Lambda_{r}$ and $\Lambda_{\theta}$, and computation time of the orbits, $\sim$ seconds, is much smaller than that of gravitational waves from EMRIs, hours to days [28, 29, 30]. Thus we believe that the analytical solutions are very useful for the computation of gravitational waves from EMRIs. In the time domain calculation of the Teukolsky formalism (see brief review in section 3.8 in [10]), we may need the inversion of $t(\lambda)$ in order to compute the orbits, $r(\lambda)$, $\cos \theta(\lambda), t(\lambda)$ and $\phi(\lambda)$, in the coordinate time. Although we do not know the analytical expression of $\lambda(t)$, we may easily obtain $\lambda(t)$ by numerical iteration if we set the initial solution as $\lambda=t / \Gamma$. Thus it may also be useful in the time domain calculation if the numerical iteration converges faster than the numerical integration of the geodesic equation.

We may also apply these solutions to investigate the properties of geodesics of Kerr black holes. Although it seems difficult to classify orbits in the strong field because of its complexities, Levin et al recently suggested a taxonomy of orbits introducing 
a rational number which is constructed from orbital frequencies [31, 32]. Both the analytical expressions of the fundamental frequencies in this paper and the taxonomy of orbits may help us to discuss the conditions characterizing zoom-whirl orbits [6] and other extreme phenomena in Kerr backgrounds. The other applications may be null or unbound geodesics. We can apply our method to them with a few modifications. For null geodesics, we have to eliminate the mass term of the small body in the geodesics. For unbound geodesics, we can express the orbits in terms of the elliptic integrals using Mino time although there are no fundamental frequencies for the orbits. However, it should be noted that we may have to improve computation time when we consider null or unbound geodesics because we have to trace the orbits for longer time than bound orbits cases. If we can not improve computation time, we may have to use both analytical solutions and numerical integration[12]. Finally, we note that we can not use the analytical solutions in this paper when the inclination angle from the equatorial plane of black hole is $\theta_{\text {inc }}=\pi / 2$. This is because $\Phi_{\theta}(\cos \theta)$ in (1) diverges when $\theta=0$, and its elliptic integral also diverges. We do not know how to address this issue without any further approximation though one can solve it if one uses a post-Newtonian expansion [22]. All of them will be discussed in a future work.

\section{Acknowledgments}

We would like to thank Bala Iyer and Hiroyuki Nakano for useful comments. W.H. was supported by the JSPS Research Fellowships for Young Scientists, No. 1756 and also supported by the 21st Century COE program "Towards a New Basic Science; Depth and Synthesis" at Osaka university from the Ministry of Education, Culture, Sports, Science and Technology of Japan.

\section{Appendix A. formulas of integrals of radial motion}

In this appendix, we derive some formulas which are needed to obtain $\Upsilon_{t^{(r)}}$ and $\Upsilon_{\theta^{(r)}}$ in section 3 and $t^{(r)}$ and $\phi^{(r)}$ in section 4. In order to compute them, we have to investigate $\int_{r_{2}}^{r} \mathrm{~d} r^{\prime} /\left\{\left(r^{\prime}-r_{ \pm}\right) \sqrt{R\left(r^{\prime}\right)}\right\}, \int_{r_{2}}^{r}\left(r^{\prime}\right)^{2} \mathrm{~d} r^{\prime} / \sqrt{R\left(r^{\prime}\right)}$ and $\int_{r_{2}}^{r} \mathrm{~d} r^{\prime} /\left\{\left(r^{\prime}-M\right)^{2} \sqrt{R\left(r^{\prime}\right)}\right\}$, see (19)). Since $\int_{r_{2}}^{r} r^{\prime} \mathrm{d} r^{\prime} / \sqrt{R\left(r^{\prime}\right)}$ is derived in section 3 and $\int_{r_{2}}^{r} \mathrm{~d} r^{\prime} /\left\{\left(r^{\prime}-M\right) \sqrt{R\left(r^{\prime}\right)}\right\}$ can be derived from $\int_{r_{2}}^{r} \mathrm{~d} r^{\prime} /\left\{\left(r^{\prime}-r_{ \pm}\right) \sqrt{R\left(r^{\prime}\right)}\right\}$ when we set $r_{ \pm}=M$, i.e. $a=M$, we do not show again these expressions in this appendix.

As we derived $\int_{r_{2}}^{r} r^{\prime} \mathrm{d} r^{\prime} / \sqrt{R\left(r^{\prime}\right)}$ in section 3, it is useful to transform $r$ into $y_{r}$. Then 
we have the following relations.

$$
\begin{aligned}
& \frac{1}{r-r_{ \pm}}=\frac{1}{r_{3}-r_{ \pm}}\left[1-\frac{r_{2}-r_{3}}{r_{2}-r_{ \pm}} \frac{1}{1-h_{ \pm} y_{r}^{2}}\right] \\
& r^{2}=r_{3}^{2}+\frac{\left(r_{2}-r_{3}\right)\left(r_{2}+3 r_{3}\right)}{2} \frac{1}{1-h_{r} y_{r}^{2}}+\frac{\left(r_{2}-r_{3}\right)^{2}}{4 h_{r}}\left[\frac{1}{\left(y_{r}-h_{r}^{-1 / 2}\right)^{2}}+\frac{1}{\left(y_{r}+h_{r}^{-1 / 2}\right)^{2}}\right], \\
& \left(\frac{r_{3}-M}{r-M}\right)^{2}=1+\frac{1}{2} \frac{r_{2}-r_{3}}{r_{2}-M}\left(\frac{r_{2}-r_{3}}{r_{2}-M}-4\right) \frac{1}{1-h_{M} y_{r}^{2}} \\
& +\frac{1}{4 h_{M}}\left(\frac{r_{2}-r_{3}}{r_{2}-M}\right)^{2}\left[\frac{1}{\left(y_{r}-h_{M}^{-1 / 2}\right)^{2}}+\frac{1}{\left(y_{r}+h_{M}^{-1 / 2}\right)^{2}}\right],
\end{aligned}
$$

where $h_{M}=h_{ \pm}(a=M)=\left(r_{1}-r_{2}\right)\left(r_{3}-M\right) /\left[\left(r_{1}-r_{3}\right)\left(r_{2}-M\right)\right]$.

Then it is straightforward to compute $\int_{r_{2}}^{r} \mathrm{~d} r^{\prime} /\left\{\left(r^{\prime}-r_{ \pm}\right) \sqrt{R\left(r^{\prime}\right)}\right\}$ as

$$
\begin{aligned}
\int_{r_{2}}^{r} \frac{\mathrm{d} r^{\prime}}{\left(r^{\prime}-r_{ \pm}\right) \sqrt{R\left(r^{\prime}\right)}}= & \frac{2}{\left(r_{3}-r_{ \pm}\right) \sqrt{\left(1-\mathcal{E}^{2}\right)\left(r_{1}-r_{3}\right)\left(r_{2}-r_{4}\right)}}\left[F\left(\arcsin y_{r}, k_{r}\right)\right. \\
& \left.-\frac{r_{2}-r_{3}}{r_{2}-r_{ \pm}} \Pi\left(\arcsin y_{r},-h_{ \pm}, k_{r}\right)\right]
\end{aligned}
$$

However, we need a reformulation of the last terms of both $\int_{r_{2}}^{r}\left(r^{\prime}\right)^{2} \mathrm{~d} r^{\prime} / \sqrt{R\left(r^{\prime}\right)}$ and $\int_{r_{2}}^{r} \mathrm{~d} r^{\prime} /\left\{\left(r^{\prime}-M\right)^{2} \sqrt{R\left(r^{\prime}\right)}\right\}$. If we set $J_{n}[c]=\int_{0}^{y} \mathrm{~d} y^{\prime} /\left\{\left(y^{\prime}-c\right)^{n} \sqrt{\varphi\left(y^{\prime}\right)}\right\}$, where $\varphi(y)=\left(1-y^{2}\right)\left(1-k_{r}^{2} y^{2}\right)$, we can represent these terms as $J_{2}[c]+J_{2}[-c]$. Using reduction formula of the elliptic integrals, see section 17.1.5 in [14], we can express $J_{2}[c]+J_{2}[-c]$ in terms of the elliptic integrals as

$$
\begin{aligned}
J_{2}[c]+J_{2}[-c]= & \frac{2}{\varphi(c)}\left\{\left[\left(2 c^{2}-1\right) k_{r}^{2}-1\right] \Pi\left(\psi,-c^{-2}, k_{r}\right)+\left(1-k_{r}^{2} c^{2}\right) F\left(\psi, k_{r}\right)-E\left(\psi, k_{r}\right)\right. \\
& \left.-\left[\frac{y \sqrt{\varphi(y)}}{y^{2}-c^{2}}\right]_{0}^{y}\right\}
\end{aligned}
$$

where $\psi=\arcsin y$.

Then we can express $\int_{r_{2}}^{r}\left(r^{\prime}\right)^{2} \mathrm{~d} r^{\prime} / \sqrt{R\left(r^{\prime}\right)}$ and $\int_{r_{2}}^{r} \mathrm{~d} r^{\prime} /\left\{\left(r^{\prime}-M\right)^{2} \sqrt{R\left(r^{\prime}\right)}\right\}$ respectively as

$$
\begin{aligned}
& \int_{r_{2}}^{r} \frac{\left(r^{\prime}\right)^{2}}{\sqrt{R\left(r^{\prime}\right)}} \mathrm{d} r^{\prime}=\frac{2}{\sqrt{\left(1-\mathcal{E}^{2}\right)\left(r_{1}-r_{3}\right)\left(r_{2}-r_{4}\right)}}\left[\frac{\left(r_{3}\left(r_{1}+r_{2}+r_{3}\right)-r_{1} r_{2}\right)}{2} F\left(\arcsin y_{r}, k_{r}\right)\right. \\
& +\frac{\left(r_{2}-r_{3}\right)\left(r_{1}+r_{2}+r_{3}+r_{4}\right)}{2} \Pi\left(\arcsin y_{r},-h_{r}, k_{r}\right) \\
& +\frac{\left(r_{1}-r_{3}\right)\left(r_{2}-r_{4}\right)}{2} E\left(\arcsin y_{r}, k_{r}\right) \\
& \left.+\frac{\left(r_{1}-r_{3}\right)\left(r_{2}-r_{4}\right)}{2} \frac{y_{r} \sqrt{\left(1-y_{r}^{2}\right)\left(1-k_{r}^{2} y_{r}^{2}\right)}}{y_{r}^{2}-h_{r}^{-1}}\right] \text {, } \\
& \int_{r_{2}}^{r} \frac{\left(r_{3}-M\right)^{2}}{\left(r^{\prime}-M\right)^{2} \sqrt{R\left(r^{\prime}\right)}} \mathrm{d} r^{\prime}=\frac{2}{\sqrt{\left(1-\mathcal{E}^{2}\right)\left(r_{1}-r_{3}\right)\left(r_{2}-r_{4}\right)}} \\
& \times\left\{\left[1-\frac{1}{2} \frac{\left(r_{1}-r_{3}\right)\left(r_{2}-r_{3}\right)}{\left(r_{1}-M\right)\left(r_{2}-M\right)}\right] F\left(\arcsin y_{r}, k_{r}\right)\right.
\end{aligned}
$$


Analytical solutions of bound timelike geodesic orbits in Kerr spacetime

$$
\begin{aligned}
& +\frac{1}{2} \frac{r_{2}-r_{3}}{r_{2}-M}\left[\frac{r_{1}-r_{3}}{r_{1}-M}+\frac{r_{2}-r_{3}}{r_{2}-M}+\frac{r_{4}-r_{3}}{r_{4}-M}-4\right] \\
& \times \Pi\left(\arcsin y_{r},-h_{M}, k_{r}\right) \\
& +\frac{1}{2} \frac{\left(r_{1}-r_{3}\right)\left(r_{2}-r_{4}\right)\left(r_{3}-M\right)}{\left(r_{1}-M\right)\left(r_{2}-M\right)\left(r_{4}-M\right)} E\left(\arcsin y_{r}, k_{r}\right) \\
& +\frac{1}{2} \frac{\left(r_{1}-r_{3}\right)\left(r_{2}-r_{4}\right)\left(r_{3}-M\right)}{\left(r_{1}-M\right)\left(r_{2}-M\right)\left(r_{4}-M\right)} \frac{y_{r} \sqrt{\left(1-y_{r}^{2}\right)\left(1-k_{r}^{2} y_{r}^{2}\right)}}{y_{r}^{2}-h_{M}^{-1}}
\end{aligned}
$$

\section{Appendix B. $|a|=M$ case}

In this appendix, we show the analytical expressions of $\Gamma, \Upsilon_{\phi} t^{(r)}$ and $\phi^{(r)}$ in the case $|a|=M$. Using the results of Appendix A, we derive them as

$$
\begin{aligned}
& \Gamma=4 M^{2} \mathcal{E}+\frac{2 a^{2} \mathcal{E} z_{+} \Upsilon_{\theta}}{\pi \mathcal{L}_{z} \sqrt{\epsilon_{0} z_{+}}}\left[K\left(k_{\theta}\right)-E\left(k_{\theta}\right)\right] \\
& +\frac{2 \Upsilon_{r}}{\pi \sqrt{\left(1-\mathcal{E}^{2}\right)\left(r_{1}-r_{3}\right)\left(r_{2}-r_{4}\right)}}\left\{\frac { \mathcal { E } } { 2 } \left[\left(r_{3}\left(r_{1}+r_{2}+r_{3}\right)-r_{1} r_{2}\right) K\left(k_{r}\right)\right.\right. \\
& +\left(r_{2}-r_{3}\right)\left(r_{1}+r_{2}+r_{3}+r_{4}\right) \Pi\left(-h_{r}, k_{r}\right) \\
& \left.+\left(r_{1}-r_{3}\right)\left(r_{2}-r_{4}\right) E\left(k_{r}\right)\right]+2 M \mathcal{E}\left[r_{3} K\left(k_{r}\right)+\left(r_{2}-r_{3}\right) \Pi\left(-h_{r}, k_{r}\right)\right] \\
& +\frac{2 M\left(4 M^{2} \mathcal{E}-a \mathcal{L}_{z}\right)}{r_{3}-M}\left[K\left(k_{r}\right)-\frac{r_{2}-r_{3}}{r_{2}-M} \Pi\left(-h_{M}, k_{r}\right)\right] \\
& +\frac{M^{2}\left(2 M^{2} \mathcal{E}-a \mathcal{L}_{z}\right)}{\left(r_{3}-M\right)^{2}}\left[\left(2-\frac{\left(r_{1}-r_{3}\right)\left(r_{2}-r_{3}\right)}{\left(r_{1}-M\right)\left(r_{2}-M\right)}\right) K\left(k_{r}\right)\right. \\
& +\frac{\left(r_{1}-r_{3}\right)\left(r_{2}-r_{4}\right)\left(r_{3}-M\right)}{\left(r_{1}-M\right)\left(r_{2}-M\right)\left(r_{4}-M\right)} E\left(k_{r}\right) \\
& \left.\left.+\frac{r_{2}-r_{3}}{r_{2}-M}\left(\frac{r_{1}-r_{3}}{r_{1}-M}+\frac{r_{2}-r_{3}}{r_{2}-M}+\frac{r_{4}-r_{3}}{r_{4}-M}-4\right) \Pi\left(-h_{M}, k_{r}\right)\right]\right\}, \\
& \Upsilon_{\phi}=\frac{2 \Upsilon_{\theta}}{\pi \sqrt{\epsilon_{0} z_{+}}} \Pi\left(-z_{-}, k_{\theta}\right)+\frac{2 a \Upsilon_{r}}{\pi \sqrt{\left(1-\mathcal{E}^{2}\right)\left(r_{1}-r_{3}\right)\left(r_{2}-r_{4}\right)}} \\
& \times\left\{\frac{2 M \mathcal{E}}{r_{3}-M}\left[K\left(k_{r}\right)-\frac{r_{2}-r_{3}}{r_{2}-M} \Pi\left(-h_{M}, k_{r}\right)\right]\right. \\
& +\frac{2 M^{2} \mathcal{E}-a \mathcal{L}_{z}}{2\left(r_{3}-M\right)^{2}}\left[\left(2-\frac{\left(r_{1}-r_{3}\right)\left(r_{2}-r_{3}\right)}{\left(r_{1}-M\right)\left(r_{2}-M\right)}\right) K\left(k_{r}\right)\right. \\
& +\frac{\left(r_{1}-r_{3}\right)\left(r_{2}-r_{4}\right)\left(r_{3}-M\right)}{\left(r_{1}-M\right)\left(r_{2}-M\right)\left(r_{4}-M\right)} E\left(k_{r}\right) \\
& +\frac{r_{2}-r_{3}}{r_{2}-M}\left(\frac{r_{1}-r_{3}}{r_{1}-M}+\frac{r_{2}-r_{3}}{r_{2}-M}+\frac{r_{4}-r_{3}}{r_{4}-M}-4\right) \\
& \left.\left.\times \Pi\left(-h_{M}, k_{r}\right)\right]\right\} \text {, }
\end{aligned}
$$




$$
\begin{aligned}
t^{(r)}= & \frac{2}{\sqrt{\left(1-\mathcal{E}^{2}\right)\left(r_{1}-r_{3}\right)\left(r_{2}-r_{4}\right)}} \\
& \times\left\{\frac { \mathcal { E } } { 2 } \left[\left(r_{2}-r_{3}\right)\left(r_{1}+r_{2}+r_{3}+r_{4}\right) \tilde{\Pi}_{r}\left(\psi_{r},-h_{r}, k_{r}\right)\right.\right. \\
& \left.+\left(r_{1}-r_{3}\right)\left(r_{2}-r_{4}\right) \tilde{E}_{r}\left(\psi_{r}, h_{r}, k_{r}\right)\right] \\
& +2 M \mathcal{E}\left(r_{2}-r_{3}\right) \tilde{\Pi}_{r}\left(\psi_{r},-h_{r}, k_{r}\right) \\
& -\frac{2 M\left(4 M^{2} \mathcal{E}-a \mathcal{L}_{z}\right)}{r_{3}-M} \frac{r_{2}-r_{3}}{r_{2}-M} \tilde{\Pi}_{r}\left(\psi_{r},-h_{M}, k_{r}\right) \\
& +\frac{M^{2}\left(2 M^{2} \mathcal{E}-a \mathcal{L}_{z}\right)}{\left(r_{3}-M\right)^{2}}\left[\frac{\left(r_{1}-r_{3}\right)\left(r_{2}-r_{4}\right)\left(r_{3}-M\right)}{\left(r_{1}-M\right)\left(r_{2}-M\right)\left(r_{4}-M\right)} \tilde{E}_{r}\left(\psi_{r}, h_{M}, k_{r}\right)\right. \\
& \left.\left.\left.+\frac{r_{2}-r_{3}}{r_{2}-r_{1}-r_{3}} \frac{r_{2}-r_{3}}{r_{1}-M}+\frac{r_{4}-r_{3}}{r_{2}-M}-4\right) \tilde{\Pi}_{r}\left(\psi_{r},-h_{M}, k_{r}\right)\right]\right\}, \\
\phi^{(r)}= & \frac{2 a}{\sqrt{\left(1-\mathcal{E}^{2}\right)\left(r_{1}-r_{3}\right)\left(r_{2}-r_{4}\right)}}\left\{-\frac{2 M \mathcal{E}}{r_{3}-M} \frac{r_{2}-r_{3}}{r_{2}-M} \tilde{\Pi}_{r}\left(\psi_{r},-h_{M}, k_{r}\right)\right. \\
& +\frac{2 M^{2} \mathcal{E}-a \mathcal{L}_{z}}{2\left(r_{3}-M\right)^{2}} \frac{\left(r_{1}-r_{3}\right)\left(r_{2}-r_{4}\right)\left(r_{3}-M\right)}{\left(r_{1}-M\right)\left(r_{2}-M\right)\left(r_{4}-M\right)} \tilde{E}_{r}\left(\psi_{r}, h_{M}, k_{r}\right) \quad(\mathrm{B} .4) \\
& \left.\left.+\frac{r_{2}-r_{3}}{r_{2}-M}\left(\frac{r_{1}-r_{3}}{r_{1}-M}+\frac{r_{2}-r_{3}}{r_{2}-M}+\frac{r_{4}-r_{3}}{r_{4}-M}-4\right) \tilde{\Pi}_{r}\left(\psi_{r},-h_{M}, k_{r}\right)\right]\right\} .
\end{aligned}
$$

\section{References}

[1] Narayan R 2005 New J. Phys. 7199

[2] Chandrasekhar S 1983 Mathematical theory of black holes (Oxford: Oxford University Press)

[3] Wilkins D C 1972 Phys. Rev. D 5814

[4] Hughes S A 2001 Phys. Rev. D 63064016

[5] Barausse E, Hughes S A and Rezzolla L 2007 Phys. Rev. D 76044007

[6] Glampedakis K and Kennefick D 2002 Phys. Rev. D 66044002

[7] Schmidt W 2002 Class. Quantum Grav. 192743

[8] Mino Y 2003 Phys. Rev. D 67084027

[9] Drasco S and Hughes S A 2004 Phys. Rev. D 69044015

[10] Glampedakis K 2005 Class. Quantum Grav. 22 S605

[11] LISA web page http://lisa.jpl.nasa.gov/

[12] Rauch K P and Blandford R D 1994 Astrophys. J. 42146

[13] Dexter J and Agol E 2009 Astrophys. J. 6961616

[14] Abramowitz M and Stegun I A (eds) 1972 Handbook of Mathematical Functions (New York: Dover)

[15] Carlson B C 1988 Math. Comp. 51267

[16] Bardeen J M 1973 Black Holes ed C DeWitt and B S DeWitt (New York: Gordon and Breach Science Publishers)

[17] Press W H, Teukolsky S A, Vetterling W T and Flannery B P 1992 Numerical Recipes in C (Cambridge: Cambridge University Press)

[18] C. F. Sopuerta and N. Yunes 2011 Phys. Rev. D 84124060

[19] Bardeen J M, Press W H and Teukolsky S A 1972 Astrophys. J. 178347

[20] Cutler C, Kennefick D and Poisson E 1994 Phys. Rev. D 503816

[21] Hughes S A 2000 Phys. Rev. D 61084004 Hughes S A 2001 Phys. Rev. D 63 049902(E) 
Hughes S A 2002 Phys. Rev. D 65 069902(E)

Hughes S A 2003 Phys. Rev. D 67 089901(E)

[22] Ganz K, Hikida W, Nakano H, Sago N and Tanaka T 2007 Prog. Theor. Phys. 1171041

[23] Kraniotis G V 2007 Class. Quantum Grav. 241775

[24] Zill D G and Carlson B C 1970 Math. Comp. 24199

[25] Teukolsky S A 1973 Astrophys. J. 185635

[26] Mino Y, Sasaki M, Shibata M, Tagoshi H and Tanaka T 1997 Prog. Theor. Phys. Suppl. 1181

[27] Sasaki M and Tagoshi H 2003 Living Rev. Relativity 66

[28] Drasco S and Hughes S A 2006 Phys. Rev. D 73024027

[29] Fujita R, Hikida W and Tagoshi H 2009 Prog. Theor. Phys. 121843

[30] Hughes S A Drasco S Flanagan E E and Franklin J 2005 Phys. Rev. Lett. 94221101

[31] Levin J and Perez-Giz G 2008 Phys. Rev. D 77103005

[32] Levin J and Grossman B 2009 Phys. Rev. D 79043016 